\title{
Rheology of Cubic Particles in a Concentrated Colloidal Dispersion Suspending Medium
}

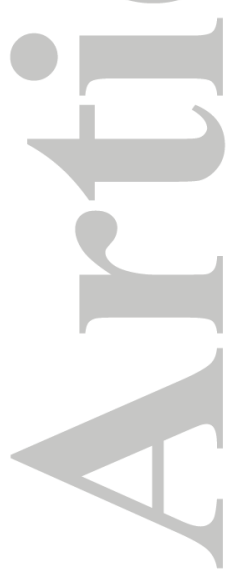

Colin D. Cwalina, Kelsey J. Harrison, and Norman J. Wagner

Department of Chemical and Biomolecular Engineering

Center for Molecular Engineering and Thermodynamics

University of Delaware

Newark, DE 19716

Revised for AIChE Journal 7/28/2016

\section{Abstract}

The flow behavior of mixtures of micron-sized cubic particles suspended in a concentrated colloidal dispersion is investigated across a broad range of cubic particle concentrations. In the semi-dilute regime, the qualitative shape of the dynamic moduli and flow curves reflect those of the underlying colloidal dispersion medium. These curves are superimposed with the underlying colloidal dispersion using shift factors that are found to be larger than those obtained in a recent study of suspensions of non-colloidal spherical particles in the same colloidal dispersion medium. At higher concentrations of cubic particles, deviations from this shifting procedure are apparent. Scaling calculations suggest depletion interactions are responsible for the increase in the low shear viscosity and confinement of the underlying

This article has been accepted for publication and undergone full peer review but has not been through the copyediting, typesetting, pagination and proofreading process which may lead to differences between this version and the Version of Record. Please cite this article as doi: 10.1002/aic. 15443

(C) 2016 American Institute of Chemical Engineers (AIChE)

Received: Jan 30, 2016; Revised: May 17, 2016; Accepted: Jun 28, 2016 
colloidal dispersion can be expected to enhance the shear thickening behavior at high shear stresses. The results of this study provide guidance for formulating suspensions through control of particle shape and mixture concentration.

\section{AREA: Transport Phenomena and Fluid Mechanics}

KEYWORDS:, Rheology, Suspensions, Colloids, Particulate Flows, Complex Fluids

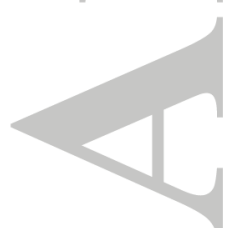

\section{Introduction}

Particle suspensions are ubiquitous across the industrial and commercial domain. In many cases, the fluids suspending the particles are non-Newtonian. Early work in this area focused on understanding the flow behavior of suspensions of spherical non-colloidal particles in polymer solutions and melts ${ }^{1-8}$. Despite the complex local flows between particles in a flowing suspension, the flow curves were found to strongly reflect the behavior of the underlying viscoelastic medium. Ohl and Gleissle ${ }^{9}$ formalized a procedure to shift the flow curves of these suspensions on to the underlying suspending fluid at constant shear stress, $\sigma$ :

$$
\text { Shift Factor }=\left.\frac{\dot{\gamma}(\phi=0)}{\dot{\gamma}(\phi)}\right|_{\sigma}=\left.\frac{\eta(\phi)}{\eta(\phi=0)}\right|_{\sigma}
$$

In the above equation, $\dot{\gamma}$ is the shear rate, $\phi$ is the particle volume fraction, and $\eta$ is the steady shear viscosity. This shifting procedure, which treats the suspending medium as a continuous fluid phase, has found remarkable success in capturing the behavior of suspension flow curves 
across a broad range of spherical particle volume fractions and various types of viscoelastic

media.

Recently, Cwalina and Wagner ${ }^{10}$ investigated suspensions comprised of non-colloidal spherical particles suspended in a concentrated colloidal dispersion. The concentrated colloidal dispersion was strongly non-Newtonian, exhibiting both shear thinning and shear thickening. It is important to note that such mixed suspensions cannot be treated simply in terms of polydispersity as the spherical non-colloidal particles and colloidal particles are separated by more than an order of magnitude in size and three orders of magnitude in mass. Suspensions comprised of mixtures of non-colloidal and colloidal particles are found in a wide variety of materials such as: concrete $^{11,12}$, asphalt ${ }^{13}$, coal water slurry fuels ${ }^{14}$, and ice cream ${ }^{15}$, just to name a few. As such, there is a significant engineering interest in understanding the flow behavior of such suspensions and how these properties can be tuned for design purposes. As many of these materials and products contain non-spherical particles, there is significant motivation for fundamental investigations of particle shape effects.

For such bimodal mixtures at volume fractions of up to $\phi=0.15$ of spherical noncolloidal particles, Cwalina and Wagner ${ }^{10}$ found the dynamic moduli and steady shear flow curves maintained the same qualitative shape of the underlying colloidal dispersion medium. The dynamic moduli curves could be shifted on to that of the neat colloidal dispersion with the shift made at constant frequency. Similar behavior has been reported with spherical particles in other viscoelastic media ${ }^{4,16-19}$. Likewise, the steady shear flow curves were successfully shifted onto the flow curve for the neat colloidal dispersion medium when compared at constant shear stress, which is consistent with the hypothesis of Ohl and Gleissle 9 . Deviations from this shifting procedure were observed at higher packing fractions of the spherical non-colloidal particles, as 
stronger shear thickening was observed. At these high packing fractions, the on-average separation distance between non-colloidal particle surfaces in suspension becomes on the order ten colloidal particle diameters, or less. Confinement is known to enhance shear thickening in colloidal dispersions ${ }^{20}$, and a recent simulation study of hydrodynamically interacting particles revealed that confinement promotes the formation of larger hydrodynamic clusters, 'hydroclusters', which leads to the observed increase in the strength of shear thickening ${ }^{21}$.

In the present work, we investigate the role of particle shape in these suspensions with a concentrated colloidal dispersion medium. The particles in this study are cubic aluminosilicate zeolites. Cwalina et al. ${ }^{22}$ recently studied the rheology of these same cubic particles suspended in a Newtonian fluid. Here, we study the effects of particle shape on mixture viscosity by suspending these cubic particles in the concentrated colloidal dispersion medium considered by Cwalina and Wagner ${ }^{10}$ in their study of suspensions comprised of mixtures of spherical noncolloidal and colloidal particles. The applicability of the shifting hypothesis of Ohl and Gleissle $^{9}$, traditionally applied to suspensions of spherical particles, is explored with regard to these suspensions of cubic particles in a non-Newtonian suspending medium. Furthermore, the possibility of enhanced confinement effects due to the shape of the non-colloidal particles is further examined by studying the mixture suspension rheology at high packing fractions of cubic particles. This study complements the existing literature on suspensions with non-Newtonian suspending fluids, and the results are of significant practical interest as they offer insight into how the flow properties of these suspensions can be tuned and engineered through the particle shape. 


\section{Experimental Section}

Materials

The primary suspending medium used in this study was a concentrated colloidal

dispersion. The dispersion consists of silica nanoparticles (Seahostar KE-P50, Nippon Shokubai Co., Tokyo, Japan) of radius $a=260 \mathrm{~nm}$ and particle density $\rho_{\mathrm{p}}=1.96 \mathrm{~g} / \mathrm{cm}^{323}$ dispersed in a near-index matching polyethylene glycol $(\mathrm{PEG})$ [average molecular weight $=200]$ suspending fluid (Aldrich Chemical Company, Allentown, PA; $\eta_{f}=0.05 \mathrm{~Pa}^{*} \mathrm{~s}$ at $25^{0} \mathrm{C}, \rho_{\mathrm{p}}=1.12 \mathrm{~g} / \mathrm{cm}^{3}$ ) at a colloid particle volume fraction of $\phi=0.40$. The volume faction is calculated using these reported densities and the sample is formulated gravimetrically. This is the identical colloidal dispersion studied by Cwalina and Wagner ${ }^{10}$ in their study of spherical non-colloidal particles in a concentrated colloidal dispersion. The colloidal dispersion was gently roll mixed for one week to insure homogeneity throughout the sample.

Suspensions were formulated by the addition of cubic aluminosilicate zeolites (Advera ${ }^{\circledR}$ 401) from PQ Corporation (Philadelphia, PA) to the colloidal dispersion medium. The SEM image in Figure 1 reveals the cubic nature of these particles, which includes facets, edges, and corners. The edge length $(l)$ distribution as reported by the manufacturer is as follows: $l_{10}=1.3$ $\mu \mathrm{m}, l_{50}=3.0 \mu \mathrm{m}, l_{90}=5.8 \mu \mathrm{m}$. The characterization of these cubic particles in a Newtonian fluid was reported recently by Cwalina et $a .^{22}$ and the suspension compositions of the present study are reported in Table 1. The volume fraction of cubic particles, $\phi_{\text {cubes, }}$, is defined as the volume of cubic particles, as calculated from the measured solution density, divided by the total suspension volume. In this manner, the ratio of the volume of KE-P50 colloidal particles to the volume of PEG-200 remains fixed for each suspension at $40 \% \mathrm{v} / \mathrm{v}$. 


\section{Rheological Characterization}

Rheological characterization was performed on an AR-2000 Rheometer (TA

Instruments, New Castle, DE) with a $40 \mathrm{~mm} 2^{0}$ cone and plate tooling. The dynamic moduli were measured during frequency sweeps at a shear stress-amplitude of $0.1 \mathrm{~Pa}$, which were performed in directions of both increasing and decreasing frequency to demonstrate reversibility. The steady shear viscosity was likewise measured with steady flow sweeps in the ascending and descending directions. All measurements in this study were performed at $25^{\circ} \mathrm{C}$. The largest particle Reynolds number, $R e_{p}$, encountered during measurement was on the order of $10^{-3}$, such that the criterion for Stokes flow was satisfied, and particle inertia can be neglected.

The steady flow curve of the primary underlying colloidal dispersion suspending medium (colloidal particle volume fraction $\phi=0.40$ ) is shown in Figure 2. The flow curve for a comparable volume fraction of cubic particles in the same Newtonian suspending fluid is shown for reference. The colloidal dispersion exhibits flow behavior characteristic of near hard-sphere dispersions. At low shear stresses, the viscosity shear thins into the high shear plateau, before ultimately increasing toward the shear-thickened state at the highest shear stresses probed. Shear thickening in colloidal dispersions is well-established to be stress-controlled ${ }^{24-26}$, and the onset of shear thickening in this dispersion occurs around $\sigma \sim 100 \mathrm{~Pa}$. Cwalina et al. $^{22}$ studied the rheology of these cubic particles in a Newtonian fluid and found shear thickening in these suspensions is likewise stress-controlled, with the onset of shear thickening around $\sigma \sim 1 \mathrm{~Pa}$, which is two orders of magnitude smaller than that of the underlying colloidal dispersion. That larger particles shear thicken at lower shear stresses is well established ${ }^{27}$, and efforts have been 
made to investigate the effects of particle shape ${ }^{28}$. This large difference in the onset stress for shear thickening will become important later on in the discussion when deciphering the origin of shear thickening in the mixed suspensions of the present study. Importantly, it should be noted that sedimentation effects associated with both the colloidal particles and the cubic particles are expected to be negligible on the timescale over which rheological measurements are performed $^{22}$.

A limited study was performed of suspensions of cubic particles in a colloidal dispersion medium where the colloidal particle volume fraction was $\phi=0.20$. These experiments were performed to assess the effect of possible depletion attractions due to the colloidal dispersion and its effect on suspension rheology. These suspension compositions are reported in Table 2.

\section{Results and Discussion}

\section{Linear Viscoelastic Regime}

The dynamic moduli curves for the suspensions of cubic particles in a concentrated colloidal dispersion suspending medium (colloidal particle volume fraction $=0.40$ ) are shown in Figure 3. Up to a volume fraction of $\phi_{\text {cubes }}=0.115$, the dynamic moduli curves exhibit the same qualitative shape as that for underlying colloidal dispersion. This behavior is qualitatively similar to that observed for suspensions of non-colloidal spheres in other types of viscoelastic media ${ }^{4,16-19}$. In this semi-dilute regime, the mixed suspensions' dynamic moduli curves can be shifted onto that for the underlying colloidal dispersion using a single shift factor that shifts the curves vertically when plotted against the frequency. This superposition of the dynamic moduli 
curves in this concentration regime is shown in Figure 4, and it can be seen that the terminal scaling of the moduli is maintained in these suspensions. The shift factors required to achieve superposition of both the storage and loss moduli are shown in Figure 5. Compared to the suspensions of spherical non-colloidal particles in the colloidal dispersion medium ${ }^{10}$, the shift factors required to superimpose the dynamic moduli curves of the suspensions of the present study are substantially larger. Furthermore, in contrast to the suspensions of spherical noncolloidal particles in a colloidal dispersion medium where the shift factors for the storage and loss moduli were essentially identical, the shift factor for the storage modulus is found to be larger than that of the loss modulus for suspensions of cubic particles in a colloidal dispersion medium.

At higher packing fractions of cubic particles, it is not possible to shift the storage moduli curves onto that of the colloidal dispersion, as there is a significant increase in the magnitude of the storage modulus at low frequencies. Interparticle attractive forces are known to lead to large increases in the storage moduli ${ }^{29}$. For the suspensions of the present study, the large increase in the storage moduli at high packing fractions and the larger storage moduli shift factors in the semi-dilute regime may be due to attractive forces between the particles themselves (such as van der Waals forces), or due to a depletion-induced attraction with the colloidal particles acting as the depletant. The possibility of depletion-induced attraction in these mixtures will be revisited later in the discussion.

In Figure 6, the purely hydrodynamic component of the loss modulus, $\eta$ ' $\omega$, is subtracted off to give only the contribution from Brownian and interparticle forces. In this manner, a characteristic relaxation time for the suspensions can be defined from the inverse of the crossover frequency, $\omega_{\text {cross-over. }}$ The cross-over frequency relative to that of the underlying colloidal 
dispersion medium is plotted in Figure 7 as a function of the volume fraction of cubic particles.

At low concentrations, the cross-over frequency is identical to that of the colloidal dispersion.

This result is consistent with that of Cwalina and Wagner ${ }^{10}$, where the addition of small amounts of spherical non-colloidal particles to the colloidal dispersion medium did not change the cross-

over frequency. However, at higher concentrations, the cross-over frequency becomes a decreasing function of the cubic particle concentration. At $\phi_{\text {cubes }}=0.329$, the cross-over frequency of the suspension is almost an order of magnitude smaller than that of the underlying colloidal dispersion.

For mixtures of non-colloidal spheres suspended in the colloidal dispersion medium, it was shown that the relative high frequency viscosity was well-described by the Einstein equation in the dilute limit ${ }^{30}$, and the expansion in particle volume fraction to order $\phi^{2}$ in the semi-dilute regime $^{31}$. These particular equations were derived for spherical particles in a Newtonian fluid, but found rather surprising applicability in suspensions of spherical particles in a non-Newtonian colloidal dispersion medium. Cwalina et $a .^{22}$ demonstrated that in the semi-dilute concentration regime (up to $\sim \phi_{\text {cubes }}=0.137$ ), the concentration-dependence of the relative viscosity of suspensions of cubic particles in a Newtonian fluid could be captured by a similar expansion in cubic particle volume fraction up to the order $\phi^{2}$ term. In Figure 8, the relative high frequency viscosity of the suspension mixtures in the present study is plotted as a function of the cubic particle volume fraction in the semi-dilute regime. Similar to what was observed for the suspensions of spherical non-colloidal particles, the relative high frequency viscosity for suspensions in a non-Newtonian colloidal dispersion medium is in good agreement with results for cubic particles suspended in a Newtonian fluid in the semi-dilute regime. 
Brownian motion in a concentrated colloidal dispersion gives rise to viscoelasticity ${ }^{32}$. A hallmark of this phenomenon in dispersions of spherical colloids is the emergence of a plateau storage modulus at high frequencies. Although a plateau modulus is not quite reached at the highest frequencies probed in this study, an estimate of the plateau modulus is taken as the value of the storage modulus at the highest frequency (a similar criterion was used by Shikata and Pearson ${ }^{32}$ in their seminal study of viscoelasticity in concentrated colloidal dispersions). As seen in Figure 9, the value of the plateau modulus relative to that of the underlying colloidal dispersion grows by almost an order of magnitude at high concentrations of cubic particles. This increase in suspension elasticity can arise either from interactions between cubic particles, which are not evident when suspended in a Newtonian fluid ${ }^{22}$, or due to confinement effects of the underlying colloidal dispersion.

Steady Shear Rheology: Semi-Dilute Regime

The steady shear viscosity for these mixed suspensions is plotted in Figure 10 as a function of the applied shear stress and volume fraction of cubic particles. As the concentration of cubic particles is increased, the flow curves maintain the same qualitative shape of the underlying colloidal dispersion, but shift vertically to higher viscosities. Similar behavior was observed by Cwalina and Wagner ${ }^{10}$ for semi-dilute suspensions of spherical non-colloidal particles in the same concentrated colloidal dispersion. In the spirit of Ohl and Gleissle's hypothesis ${ }^{9}$, a shift factor was proposed to superimpose the suspension flow curves on to that of the underlying colloidal dispersion medium with a single shift factor. This approach required treating the colloidal dispersion as a single continuous fluid phase. As seen in Figure 11, a successful superposition of the viscosity data is indeed achieved with a single shift factor, demonstrating that the shifting procedure of Ohl and Gleissle ${ }^{9}$ is applicable to this class of 
suspensions with cubic particles suspended in a non-Newtonian fluid medium that exhibits both shear thinning and shear thickening. This result is quantitatively consistent with recent observations for mixtures with spherical non-colloidal particles suspended in the same colloidal dispersion ${ }^{10}$, and with models proposed for bimodal suspensions such as in coal-water slurries ${ }^{33}$.

The shift factor required to superimpose a suspension's viscosity curve onto that of the underlying colloidal dispersion medium is essentially a relative viscosity - the suspension viscosity divided by the suspending medium viscosity. The relative viscosity of the suspensions of cubic particles suspended in the concentrated colloidal dispersion medium is shown in Figure 12. Also plotted in this figure are the results obtained by Cwalina et al. ${ }^{22}$ for the cubic particles in a Newtonian suspending fluid in the semi-dilute regime. The measured relative steady shear viscosities of the present suspensions are slightly larger than the expectations for cubic particles in a Newtonian fluid. A similar result was observed for the related system of bimodal suspensions of spherical particles by Cwalina and Wagner ${ }^{10}$, where the relative steady shear viscosity of suspensions of spherical non-colloidal particles in the same colloidal dispersion medium was slightly larger than predictions for spherical particles in a Newtonian fluid. It should be noted that this phenomenon has been observed more broadly for spherical particles in other types of viscoelastic media as well, and this behavior remains unexplained ${ }^{19,34,35}$.

Due to the possible confining effects afforded by the flat surfaces of the cubic particles on the flow of the colloidal suspension, we consider the possible effects of such confinement on the viscosity of the underlying colloidal dispersion. Swan and Brady ${ }^{36}$ showed that confinement of a colloidal dispersion to length scales of order ten particle radii or less led to an increase in the hydrodynamic viscosity. Physically, this enhanced viscosity comes from interactions between a colloidal particle and the boundary as well as increased hydrodynamic interactions between 
colloidal particles themselves. While the exact orientation of cubic particles with respect to one another under steady shear is expected to be somewhat random, it is anticipated that there will be configurations where cubic particle faces are oriented parallel to one another. In such an instance, the colloidal particles are confined locally between the much larger faces of the cubic particle, and the calculations of Swan and $\operatorname{Brady}^{36}$ can be used to estimate the viscosity increase of the underlying colloidal dispersion medium.

A geometric model can be used to predict the on-average surface separation distance between cubic particles ${ }^{24,37}$ :

$$
\frac{h_{\text {cubes }}}{l}=\left[\left(\frac{\phi_{\text {max }}}{\phi_{\text {cubes }}}\right)^{1 / 3}-1\right]
$$

where $h_{\text {cubes }}$ is the on-average surface separation distance between cubic particles, $l$ is the average cube edge length, and $\phi_{\max }$ is the maximum packing fraction of cubic particles under steady shear, determined by Cwalina et al. ${ }^{22}$ to be 0.69 . At $\phi_{\text {cubes }}=0.076$, the on-average surface separation distance between cubic particles is approximately 12 colloidal particle radii, which is around the characteristic channel spacing where confinement begins to enhance the dispersion viscosity according to the calculations of Swan and Brady ${ }^{36}$. Thus, as confinement effects become more important and lead to an increase in the viscosity of the underlying colloidal dispersion medium, the suspension's relative viscosity can be calculated using the higher viscosity of the confined colloidal dispersion. When confinement effects are considered in this manner for the data in Figure 12, the relative suspension viscosity more closely follows the fit from Cwalina et $a l .{ }^{22}$ for cubic particles in a Newtonian fluid. These measurements and calculations suggest that confinement effects may become important in these mixed suspensions when the colloidal dispersion is confined between cubic particle faces at separation distances 
spanning only a few colloidal particle radii. Future simulations of these suspensions of cubic particles in a colloidal dispersion under steady shear would be useful for validating or refuting the hypothesis that confinement leads to the observed concentration dependence of the suspension relative viscosity in Figure 12

\section{Steady Shear Rheology: Concentrated Suspensions}

Figure 13 reports the steady shear viscosity as a function of the shear stress and shear rate for concentrated suspensions of cubic particles in a colloidal dispersion suspending medium. Above $\phi_{\text {cubes }}=0.115$, the qualitative shape of the flow curves deviates significantly from that of the underlying colloidal dispersion and the semi-dilute suspensions at both low and high shear rates (stresses).

Above $\phi_{\text {cubes }}=0.115$, at the lowest measured shear stresses, the steady shear viscosity does not tend toward a zero-shear viscosity and the magnitude of the low shear viscosity rises by several orders of magnitude. Note that this deviation in behavior cannot simply be due to the hydrodynamic confinement effect explored in the previous section, as that would result in a vertical shift in the viscosity. Consequently, a weak, interparticle force acting between cubic particles is suspected to be the source of this enhanced low shear viscosity. A logical candidate is depletion attractions ${ }^{38}$ resulting from the excluded volume, with regards to the colloidal particles, due to close approach of the cubic particles, as illustrated in Figure 14. The maximum possible stress from depletion attractions will occur when the cube faces are oriented parallel and aligned with one another. In this configuration, the overlap of excluded volume is maximized.

The depletion potential between the two cubic particles, $U^{d e p}$, scales with the product of the osmotic pressure, $\pi$, and the overlap of excluded volume, $\Delta V^{39}$ : 


$$
U^{d e p} \sim \pi \Delta V
$$

For cubic particles with edge length, $L$, and spherical colloids of diameter, $d$, the maximum overlap of excluded volume can be computed as:

$$
\Delta V=2 d(L+2 d)^{2}
$$

For the suspensions in this study with cubic particles of average edge length, $L=3 \mu \mathrm{m}$, and colloidal particles of diameter, $d=520 \mathrm{~nm}$, the overlap of excluded volume is calculated to be $\Delta V \sim 4 \times 10^{-18} \mathrm{~m}^{3}$. The osmotic pressure of the colloidal dispersion is calculated using the empirical Carnahan-Starling equation of state for hard-sphere fluids ${ }^{40}$ :

$$
\frac{\pi}{n k T}=\frac{1+\phi+\phi^{2}-\phi^{3}}{(1-\phi)^{3}}
$$

For the $\phi=0.40$ colloidal dispersion, $\frac{\pi}{n k T} \sim 7$, and the maximum depletion potential between

two face-to-face aligned cubes is approximately, $\frac{U^{d e p}}{k T} \sim 150$. This would be an extremely strong attractive potential between cubic particles, but it should be viewed within the context of being the maximum possible magnitude of the potential given that the calculation is for cube faces that are perfectly aligned. Any other relative orientation of two cubic particles will result in a smaller overlap of excluded volume. A characteristic stress from these depletion attractions, $\sigma^{d e p}$, can be estimated from the product of the energy per interaction $\left(U^{d e p}\right)$ and the number of interactions in suspension, the latter quantity being proportional to the number density of cubic particles, $n_{\text {cubes }}$, such that:

$$
\sigma^{d e p} \sim U^{d e p} n_{c u b e s}
$$


Thus, for the largest concentration of cubic particles studied, the estimated stress from depletion attractions is of order $\sigma^{d e p} \sim 10^{-2} \mathrm{~Pa}$. The lowest shear stress probed during rheological measurement was comparable $\left(\sigma=10^{-2} \mathrm{~Pa}\right)$, and although there is no clear rheological signature of a yield stress, the viscosity does begin to rapidly rise on approach to this lower shear stress limit. Further, this effect is more evident for the larger concentrations of cubic particles, in agreement with expectations based on Eqn. (6). These quantitative estimates show it is indeed plausible that the relatively large increase in the low shear viscosity of these mixed suspensions can be attributed to depletion attractions between cubic particles due to exclusion of the colloidal particles. Similar calculations show such depletion effects are much less significant for mixtures of spherical particles of comparable size ratio ${ }^{10}$.

In Cwalina and Wagner's study ${ }^{10}$ of mixtures of spherical non-colloidal particles in a concentrated colloidal dispersion at high packing fractions, deviations from the shifting procedure of Ohl and Gleissle ${ }^{9}$ were identified at higher shear stresses (much larger than the characteristic depletion stress). Confinement of the colloidal fluid between spherical noncolloidal particle surfaces was shown to increase the shear thickening power law exponent, which is qualitatively consistent with previous experimental ${ }^{20}$ and simulation ${ }^{21}$ studies. The suspensions consisting of cubic particles in a concentrated colloidal dispersion medium exhibit an even more complex behavior. As seen in Figure 13, at $\phi_{\text {cubes }}=0.284$, the high shear plateau characteristic to the underlying colloidal dispersion and the semi-dilute suspensions begins to disappear. In the plot of viscosity versus shear rate, it is easy to identify two distinct shear thickening transitions marked by the orange arrows. When viewed on a plot of viscosity versus shear stress, it is seen that the onset shear stresses for the two regimes are $\sigma \sim 1 \mathrm{~Pa}$ and $\sigma \sim 100$ Pa. The latter onset stress corresponds to shear thickening of the underlying colloidal dispersion. 
The former corresponds to the onset stress for shear thickening of the cubic particles in a Newtonian fluid that was identified by Cwalina et $a l .{ }^{22}$ This is, to the best of the authors' knowledge, the first documented case of a complex fluid exhibiting multiple, distinct shear thickening transitions under steady shear. At $\phi_{\text {cubes }}=0.329$, there is only one identifiable shear thickening transition due to the cubic particles that has an onset stress of $\sigma \sim 1 \mathrm{~Pa}$. Shear thickening of the colloidal dispersion may still be present at high stresses, but its contribution to the stress is likely masked by that of cubic particles, which have a larger contribution to the overall increase in shear viscosity.

Cwalina and Wagner ${ }^{10}$ observed that confinement of the colloidal fluid between surfaces of non-colloidal spheres at high shear stresses led to an increase in the shear thickening power law exponent. Here, the power law exponent from $\sigma=100$ to $400 \mathrm{~Pa}$ (same shear stress range considered by Cwalina and Wagner ${ }^{10}$ ) is examined as a function of the volume fraction of cubic particles in suspension. Only volume fractions up to $\phi_{\text {cubes }}=0.284$ are considered, as shear thickening in this stress range for the $\phi_{\text {cubes }}=0.329$ suspension reflects shear thickening from the cubic particles themselves. The shear thickening power law exponent is plotted as a function of particle volume fraction in Figure 15 for the suspensions considered in this study and those from Cwalina and Wagner ${ }^{10}$ containing spherical non-colloidal particles suspended in the same concentrated colloidal dispersion medium.

A more revealing plot is evident when the shear thickening power law exponent plotted against the on-average surface separation distance between particles in suspension, which can be estimated from Equation 2. This analysis was already conducted previously for the spherical non-colloidal particles in the same colloidal dispersion medium ${ }^{10}$. The shear thickening power law exponent is replotted in Figure 16 as a function of $h / 2 a_{\text {colloids. }}$ This parameter physically 
represents the number of colloidal particles that could span the characteristic gap between either spherical or cubic particle surfaces in suspension.

Rather remarkably, the two data sets nearly overlap at small values of $h / 2 a_{\text {colloids. }}$ To understand this result, it is useful to consider a to-scale representation of $520 \mathrm{~nm}$ colloidal spheres, $3 \mu \mathrm{m}$ edge length cubes, and $10 \mu \mathrm{m}$ spheres as shown in Figure 17. From the perspective of the colloidal particles, the amount of confinement produced by the surface of 3 $\mu \mathrm{m}$ edge length cubes is roughly the same as that produced by $10 \mu \mathrm{m}$ diameter spheres. This result is further evidence to support the confinement hypothesis introduced by Cwalina and Wagner ${ }^{10}$. Additionally, it demonstrates the need to consider not just the particle shape, but also the particle size when assessing the effects of confinement on the shear thickening power law exponent in suspensions with a concentrated colloidal dispersion suspending medium. Swan and Brady $^{36}$, in their study of hydrodynamic effects for dispersions under confinement, did not consider shear flows leading to shear thickening, but one could expect that the increase in hydrodynamic interactions between the colloidal particles in the presence of the confining surfaces would promote stronger shear thickening.

Effect of the Colloid Dispersion Medium Composition

The effect of the colloidal dispersion medium composition was investigated through measurements of suspensions of cubic particles suspended in a colloidal dispersion with a colloidal particle volume fraction of $\phi=0.20$. As seen in Figure 18, the rheology of this $\phi=$ 0.20 colloidal dispersion is only weakly non-Newtonian at this colloidal particle concentration, with a weak shear thickening transition around $\sigma \sim 100 \mathrm{~Pa}$. 
At $\phi_{\text {cubes }}=0.203$, shear thickening is observed at an onset shear stress of $\sigma \sim 1 \mathrm{~Pa}$, which corresponds to shear thickening of the cubic particles reported by Cwalina et al. ${ }^{22}$ This shear thickening becomes much more pronounced with increasing cubic particle volume fraction. Analysis of the local derivative in Figure $18^{41}$ reveals that above $\phi_{\text {cubes }}=0.203$ there is no obvious evidence of a second shear thickening transition due to thickening of the spherical colloids at higher stresses. Importantly, the shear viscosity for the mixed suspensions at high stresses is fundamentally different. Unlike the behavior shown in Figure 13, now a second shear thinning regime is encountered that is characteristic of elastohydrodynamic effects observed for the cubic particles suspended in a Newtonian fluid ${ }^{22}$. This stark difference in behavior at high shear stresses is perhaps the most significant evidence that confinement effects dominate the mixed suspensions in the more concentrated colloidal dispersion in the shear thickening regime.

As noted, any depletion interactions in the mixed suspensions will depend on the concentration of the suspending colloidal dispersion medium. In contrast to the suspensions in the $\phi=0.40$ colloidal dispersion medium, there is no significant increase in the magnitude of the low shear viscosity in concentrated suspensions of cubic particles in the $\phi=0.20$ colloidal dispersion medium. From Eqn. (5), the osmotic pressure in the $\phi=0.20$ colloidal dispersion is calculated to be, $\frac{\pi}{n k T} \sim 2.4$, which leads to a maximum depletion potential between cubic particles of, $\frac{U^{d e p}}{k T} \sim 26$. The maximum characteristic stress from this depletion potential at the largest concentration of cubic particles considered in this section is calculated from Eqn. (6) to be $\sigma^{\text {dep }} \sim 10^{-3} \mathrm{~Pa}$. This characteristic stress is an order of magnitude smaller than that calculated for the cubic particle suspensions in the $\phi=0.40$ colloidal dispersion medium. Thus, the nearly Newtonian rheology at low shear stresses for the suspensions in the $\phi=0.20$ colloidal dispersion 
medium is consistent with calculation of depletion attraction strength, further supporting the supposition that the enhancement in low shear viscosity in the mixtures arises from depletion attractions specific to the geometry of the cubic particles.

\section{Constant Total Solids Volume Fraction}

We conclude the discussion with an examination of how the rheology of suspensions at nearly constant total solids fraction $\left(\phi_{\text {Total Solids }}=\phi_{\text {cubes }}+\phi_{K E-P 50 \text { Colloids }}\right)$ can be vastly different depending upon the relative ratios of cubes and spherical colloids in suspension. The suspension viscosity is shown in Figure 19 as a function of the shear stress for suspensions of cubic particles and spherical colloids at nearly identical total solids volume fraction. For these suspensions, the magnitude of the viscosity, even at equivalent total solids loadings, is a function of the ratio of cubic particles to spherical colloids and also a function of the applied shear stress. Clearly, the dependence is not simply monotonic with regard to either variable. This comparison suggests a methodology for mixing particles of varying shape and size to tailor the viscosity response to varying levels of applied stresses. A more detailed study would be necessary to map out this highly nonlinear mixing effect, but the results of this study provide guidance based on how the basic physical interactions, hydrodynamic and depletion, are influenced by the particle concentrations.

\section{Conclusions}

The rheology of suspension mixtures consisting of cubic particles suspended in a concentrated colloidal dispersion medium was investigated and compared and contrasted with prior work in literature for similar mixtures comprised of spherical non-colloidal particles suspended in the same colloidal dispersion medium. For semi-dilute suspensions, the dynamic 
moduli and the steady shear flow curves could be shifted onto those of the underlying colloidal dispersion medium using simple shift factors following the hypothesis of Ohl and Gleissle ${ }^{9}$. The magnitude of the shift factors required to achieve a superposition of the dynamic moduli and steady shear viscosity curves were larger than those for suspensions of spherical non-colloidal particles in the same concentrated colloidal dispersion medium ${ }^{10}$. Particle shape is known to be an important factor in determining the rheology of suspensions in Newtonian fluids, and clearly particle shape significantly alters the rheology of suspensions in a non-Newtonian colloidal dispersion medium. A possible explanation for this effect is an increase in hydrodynamic interactions due to confinement and a limiting expectation for this behavior can be obtained from recent theoretical work by Swan and Brady ${ }^{36}$.

At higher concentrations of cubic particles in the colloidal dispersion medium, the characteristic relaxation time of these suspension increases along with the plateau storage modulus. Under steady shear, two distinct shear thickening transitions emerge that reflect shear thickening of both the cubic particles and the spherical colloids. The shear thickening power law exponent corresponding to the thickening of the spherical colloids is shown to increase with the packing fraction of cubic particles, similar to the behavior observed by Cwalina and Wagner ${ }^{10}$ for suspensions of non-colloidal spheres in a concentrated colloidal dispersion. These results show that the enhanced shear thickening at high stresses due to confinement of the colloidal fluid at high particle packing fractions appears to be a universal phenomenon, and the increase in the shear thickening power law exponent can be understood in terms of the confining particle shape and size.

At higher concentrations of cubic particles in a concentrated colloidal dispersion medium, a large increase in the magnitude of the low shear viscosity is observed. Scaling calculations 
identify depletion attractions as a possible source, where exclusion of the colloidal particles upon close approach of the cubic particles can generate large interparticle forces that translate to stresses of the correct order of magnitude. The contribution of these depletion attractions to the suspension stress was found to diminish as the colloidal particle concentration of the suspending medium was reduced. Finally, limited comparison of mixed suspensions of varying colloidal particle and cube concentrations shows a rich, nonlinear behavior that affords an interesting opportunity to tune the response of colloidal dispersions for specific applications.

\section{Acknowledgment}

This work was supported by a NASA EPSCoR Grant (NNX11AQ28A) and a Delaware Space Grant Graduate Fellowship (NNX10AN63H). PQ Corporation is acknowledged for providing the cubic aluminosilicate zeolite particles.

\section{Literature Cited}

1. Highgate DJ, Whorlow RW. Rheological properties of suspensions of spheres in nonNewtonian media. Rheol Acta. 1970;9:569-576.

2. Mewis J, de Bleyser R. Concentration effects in viscoelastic dispersions. Rheol Acta. $1975 ; 14: 721-728$.

3. Kataoka T, Kitano T, Sasahara M, Nishijima K. Viscosity of particle filled polymer melts. Rheol Acta. 1978;17:149-155. 
4. Chan D, Powell RL. Rheology of Suspensions of Spherical-Particles in a Newtonian and a Non-Newtonian Fluid. J Non-Newton Fluid. 1984;15(2):165-179.

5. Metzner AB. Rheology of Suspensions in Polymeric Liquids. J Rheol. Dec $1985 ; 29(6): 739-775$.

6. Poslinski AJ, Ryan ME, Gupta RK, Seshadri SG, Frechette FJ. Rheological Behavior of Filled Polymeric Systems .1. Yield Stress and Shear-Thinning Effects. J Rheol. Oct $1988 ; 32(7): 703-735$.

7. Aral BK, Kalyon DM. Viscoelastic material functions of noncolloidal suspensions with spherical particles. J Rheol. May-Jun 1997;41(3):599-620.

8. Zarraga IE, Hill DA, Leighton DT. Normal stresses and free surface deformation in concentrated suspensions of noncolloidal spheres in a viscoelastic fluid. J Rheol. Sep-Oct 2001;45(5):1065-1084.

9. Ohl N, Gleissle W. The Characterization of the Steady-State Shear and Normal Stress Functions of Highly Concentrated Suspensions Formulated with Viscoelastic Liquids. $J$ Rheol. Mar-Apr 1993;37(2):381-406.

10. Cwalina CD, Wagner NJ. Rheology of non-Brownian particles suspended in concentrated colloidal dispersions at low particle Reynolds number. J Rheol. 2016;60:47-59.

11. Aitcin PC. Cements of yesterday and today - Concrete of tomorrow. Cement Concrete Res. Sep 2000;30(9):1349-1359.

12. Toussaint F, Roy C, Jezequel PH. Reducing shear thickening of cement-based Suspensions. Rheologica Acta. Oct 2009;48(8):883-895.

13. Zapata P, Gambatese JA. Energy Consumption of Asphalt and Reinforced Concrete Pavement Materials and Construction. Journal of Infrastructure Systems. 2005;11:9-20. 
14. Mishra SK, Senapati PK, Panda D. Rheological behavior of coal-water slurry. Energ Source. Feb 2002;24(2):159-167.

15. Goff HD. Colloidal aspects of ice cream - A review. Int Dairy J. Jun-Jul 1997;7(67):363-373.

16. Schaink HM, Slot JJM, Jongschaap RJJ, Mellema J. The rheology of systems containing rigid spheres suspended in both viscous and viscoelastic media, studied by Stokesian dynamics simulations. $J$ Rheol. May-Jun 2000;44(3):473-498.

17. See H, Jiang P, Phan-Thien N. Concentration dependence of the linear viscoelastic properties of particle suspensions. Rheologica Acta. Mar 2000;39(2):131-137.

18. Le Meins JF, Moldenaers P, Mewis J. Suspensions in polymer melts. 1. Effect of particle size on the shear flow behavior. Ind Eng Chem Res. Dec 11 2002;41(25):6297-6304.

19. Pasquino R, Grizzuti N, Maffettone PL, Greco F. Rheology of dilute and semidilute noncolloidal hard sphere suspensions. J Rheol. Nov-Dec 2008;52(6):1369-1384.

20. Chow MK, Zukoski CF. Gap Size and Shear History Dependencies in Shear Thickening of a Suspension Ordered at Rest. J Rheol. Jan-Feb 1995;39(1):15-32.

21. Bian $X$, Litvinov S, Ellero M, Wagner NJ. Hydrodynamic shear thickening of particulate suspension under confinement. J Non-Newton Fluid. 2014;213:39-49.

22. Cwalina CD, Harrison KJ, Wagner NJ. Rheology of Cubic Particles Suspended in a Newtonian Fluid. Soft Matter. 2016;published online 18 Apr. 2016.

23. Kalman DP. Microstructure and Rheology of Concetrated Suspensions of Near HardSphere Colloids, University of Delaware; 2010.

24. Bender J, Wagner NJ. Reversible shear thickening in monodisperse and bidisperse colloidal dispersions. J Rheol. Sep-Oct 1996;40(5):899-916. 
25. Maranzano BJ, Wagner NJ. The effects of particle-size on reversible shear thickening of concentrated colloidal dispersions. J Chem Phys. Jun 15 2001;114(23):10514-10527.

26. Shenoy SS, Wagner NJ. Influence of medium viscosity and adsorbed polymer on the reversible shear thickening transition in concentrated colloidal dispersions. Rheologica Acta. Apr 2005;44(4):360-371.

27. Maranzano BJ, Wagner NJ. The effects of interparticle interactions and particle size on reversible shear thickening: Hard-sphere colloidal dispersions. J Rheol. $2001 ; 45(5): 1205-12222$.

28. Egres RG, Wagner NJ. The rheology and microstructure of acicular precipitated calcium carbonate colloidal suspensions through the shear thickening transition. Journal of Rheology (1978-present). 2005;49(3):719-746.

29. Rueb CJ, Zukoski CF. Rheology of suspensions of weakly attractive particles: Approach to gelation. J Rheol. 1998;42:1451-1476.

30. Einstein A. Berichtigung zu meiner Arbeit:,,Eine neue Bestimmung der Moleküldimensionen” . Annalen der Physik. 1911;339(3):591-592.

31. Wagner NJ, Woutersen ATJM. The Viscosity of Bimodal and Polydisperse Suspensions of Hard-Spheres in the Dilute Limit. J Fluid Mech. Nov 10 1994;278:267-287.

32. Shikata T, Pearson DS. Viscoelastic Behavior of Concentrated Spherical Suspensions. $J$ Rheol. May-Jun 1994;38(3):601-616.

33. Probstein RF, Sengun MZ, Tseng TC. Bimodal model of concentrated suspension viscosity for distributed particle sizes. J Rheol. 1994;38(4):811-829.

34. Mall-Gleissle SE, Gleissle W, McKinley GH, Buggisch H. The normal stress behaviour of suspensions with viscoelastic matrix fluids. Rheologica Acta. Jan 2002;41(1-2):61-76. 
35. Tanner RI, Dai SC, Qi FZ, Housiadas K. Viscometric functions of semi-dilute noncolloidal suspensions of spheres in a viscoelastic matrix. J Non-Newton Fluid. Nov $2013 ; 201: 130-134$.

36. Swan JW, Brady JF. The hydrodynamics of confined dispersions. J Fluid Mech. Nov 2011;687:254-299.

37. Boersma WH, Laven J, Stein HN. Shear Thickening (Dilatancy) in Concentrated Dispersions. Aiche J. Mar 1990;36(3):321-332.

38. Asakura S, Oosawa F. Interaction between particles suspended in solutions of macromolecules Journal of Polymer Science. 1958;33:183-192.

39. Rossi L, Sacanna S, Irvine WTM, Chaikin PM, Pine DJ, Philipse AP. Cubic crystals from cubic colloids. Soft Matter. 2011;7(9):4139-4142.

40. Carnahan NF, Starling KE. Equation of state for noninteracting rigid spheres. J Chem Phys. 1969;51(2):635.

41. Cwalina CD. Shear Thickening Fluids for Enhanced Protection from Micrometeoroids and Orbital Debris, University of Delaware; 2016.

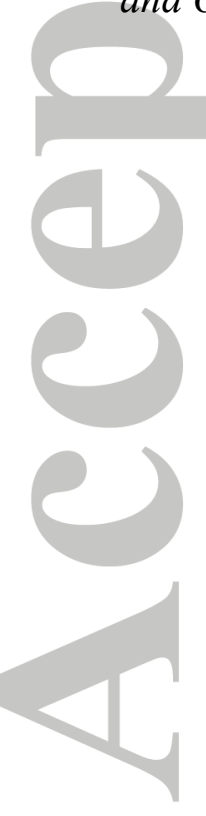



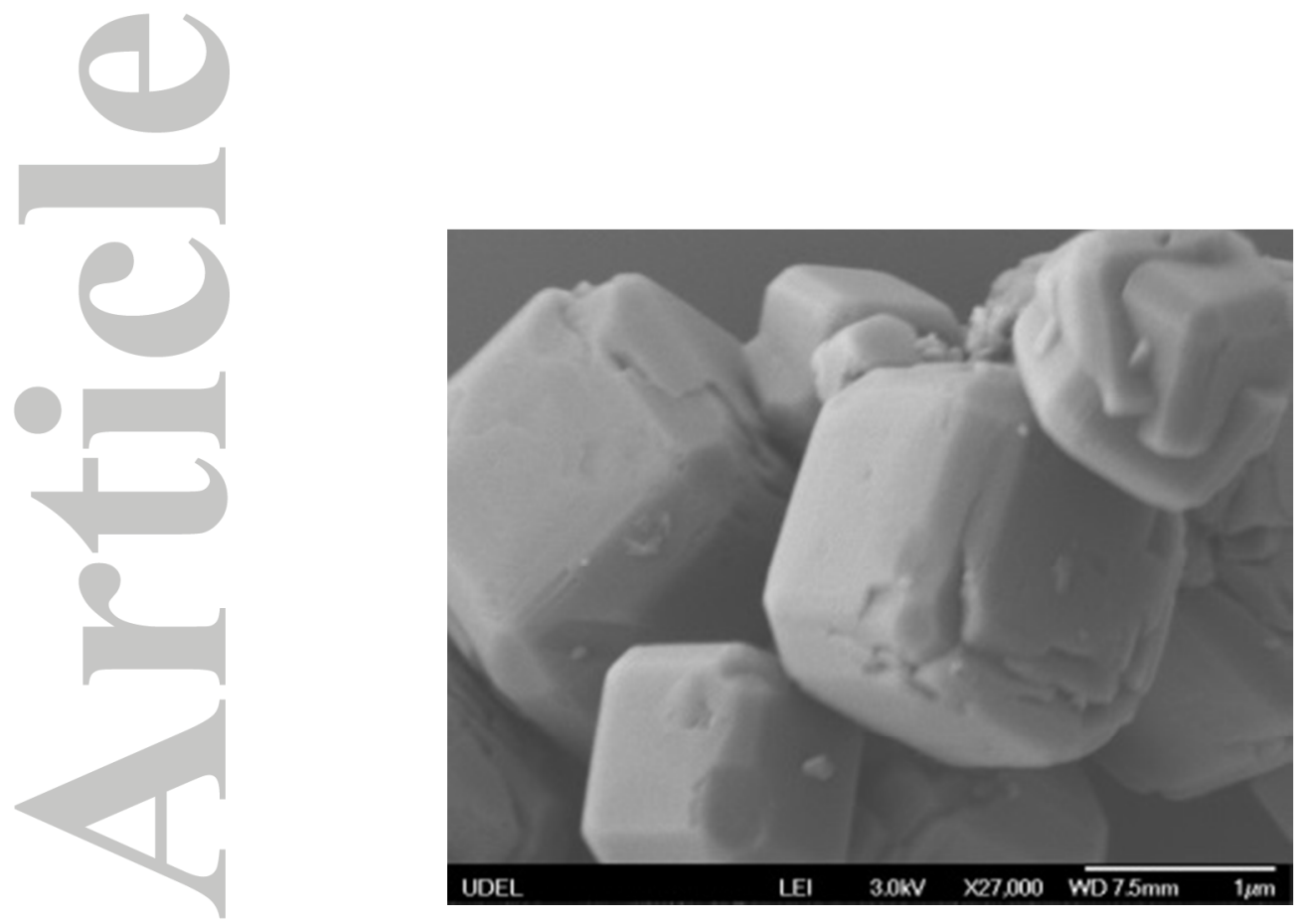

Figure 1. SEM image of cubic aluminosilicate zeolites used in this study. Image used with permission from Cwalina et al. ${ }^{22}$

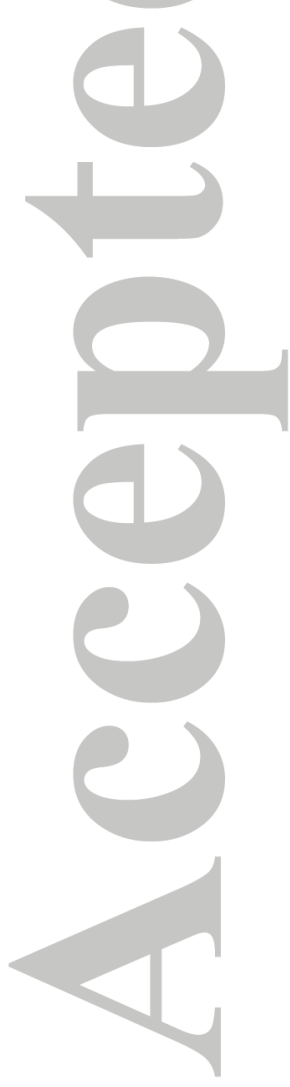



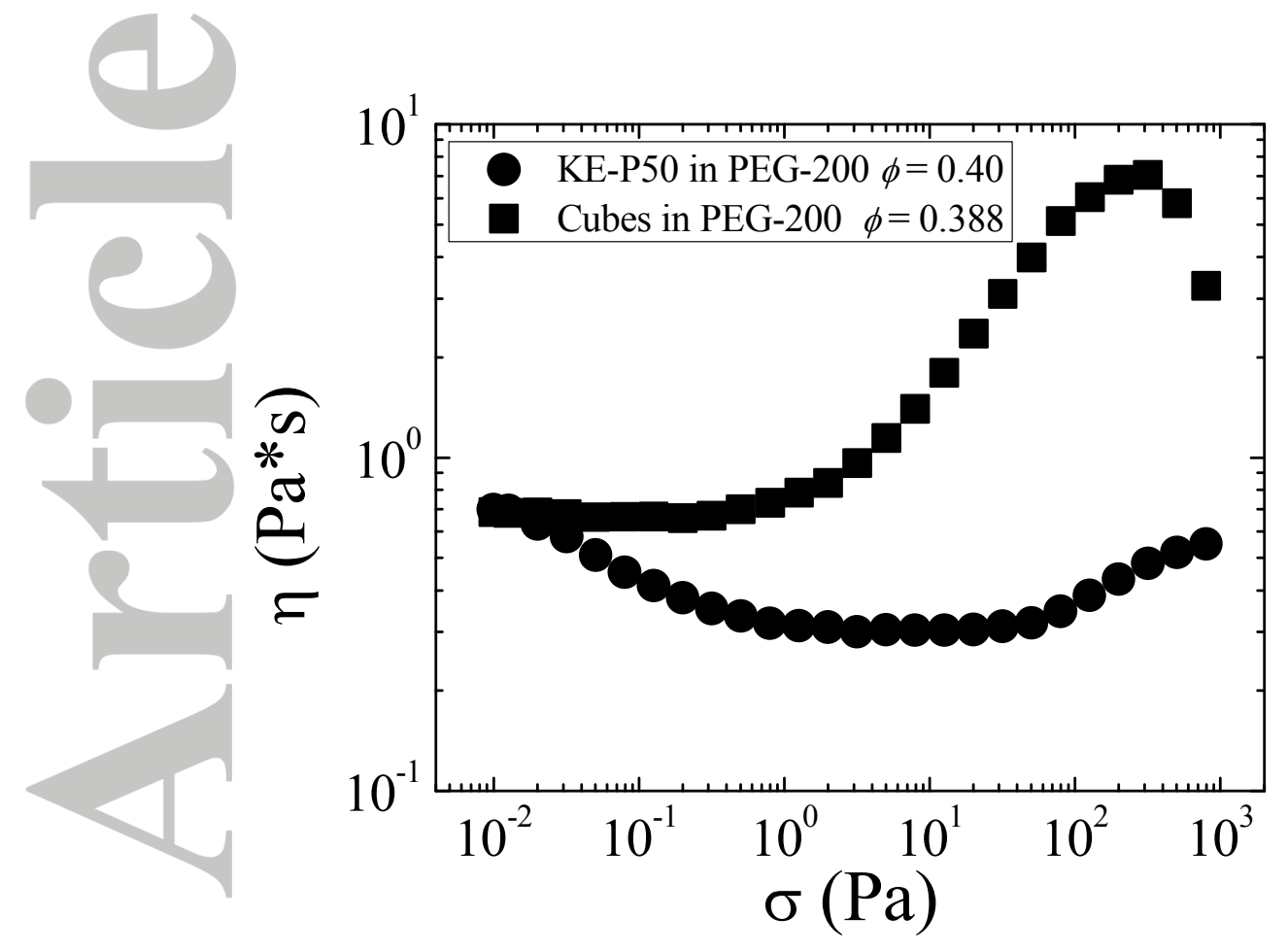

Figure 2. Steady shear viscosity of the $\phi=0.40$ colloidal dispersion suspending medium (circles) and a $\phi=0.388$ suspension of cubic particles in a Newtonian suspending medium (squares) [from Cwalina et al. ${ }^{22}$ ] plotted as a function of the applied shear stress. The onset of shear thickening in the suspension of cubic particles in a Newtonian fluid occurs around $\sigma \sim 1$ $\mathrm{Pa}$, while that for the colloidal dispersions is around $100 \mathrm{~Pa}$.

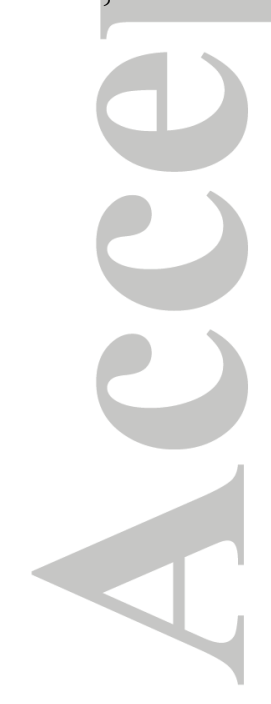




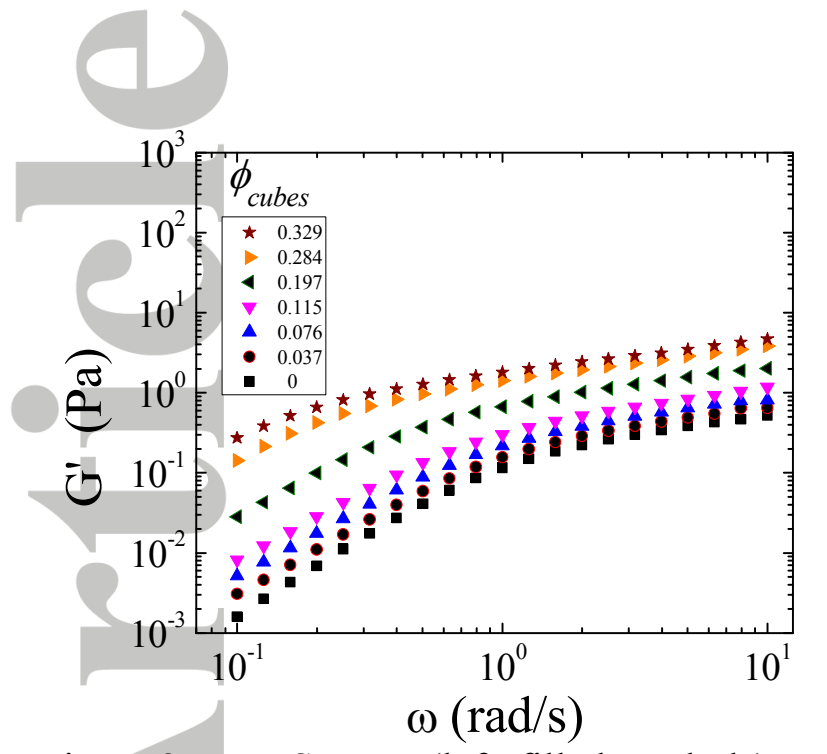

Figure 3. Storage (left, filled symbols) and

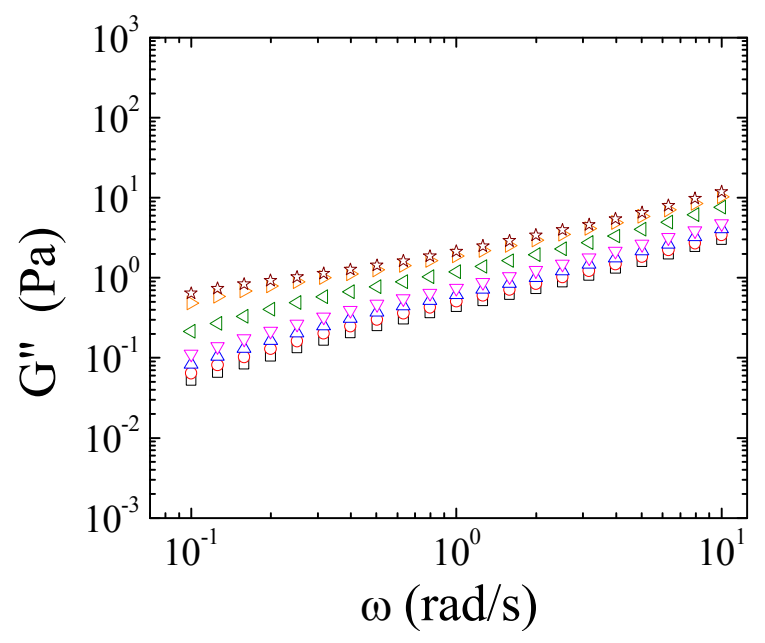

loss (right, open symbols) moduli for suspensions of cubic particles in a concentrated colloidal dispersion medium for a range of cubic particle volume fractions.
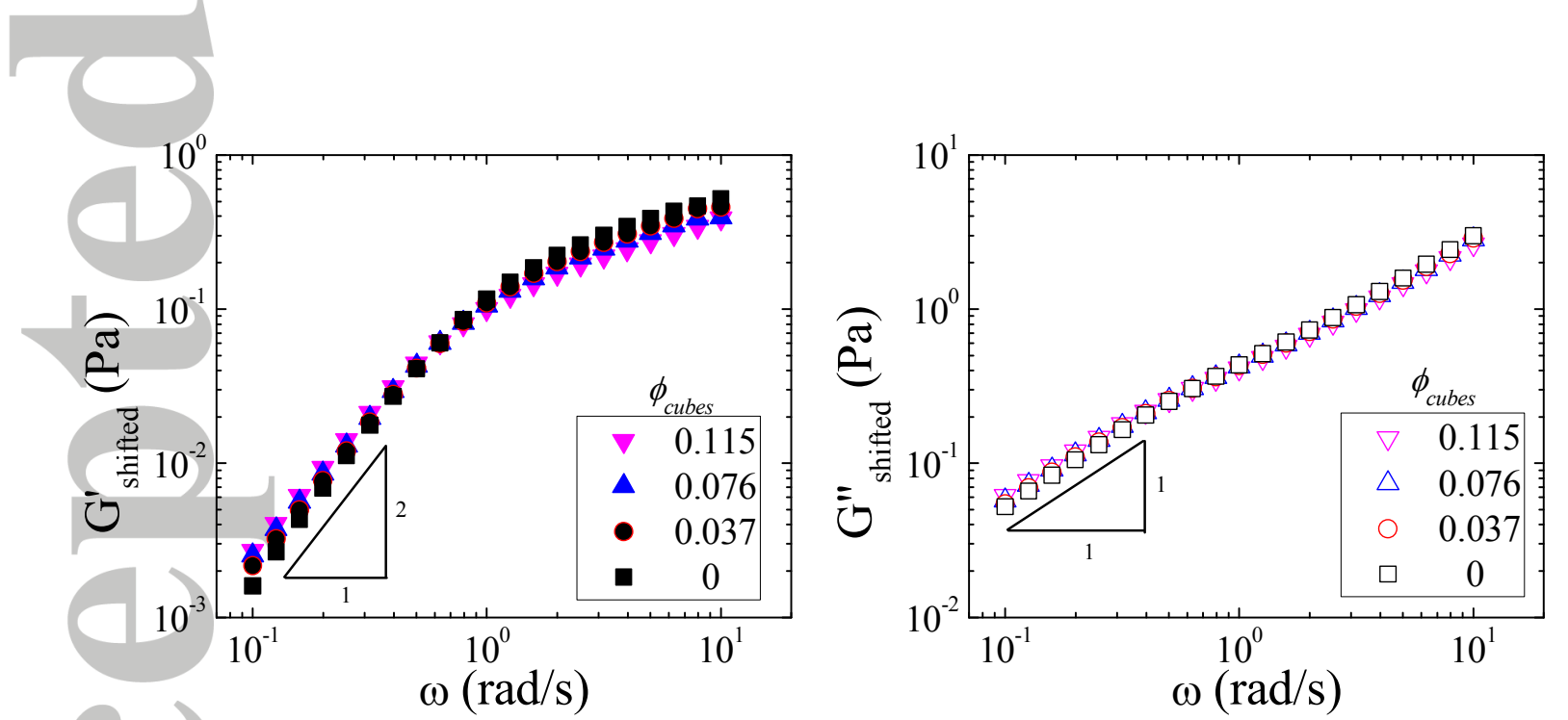

Figure 4. Suspension storage (left) and loss (right) moduli shifted onto that of the underlying colloidal dispersion medium in the semi-dilute cubic particle concentration regime. 

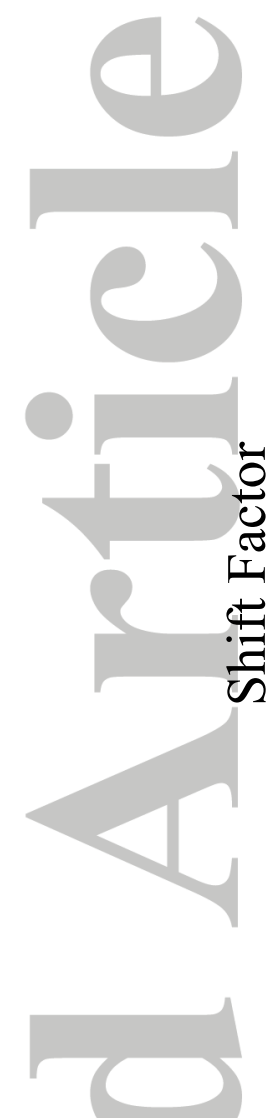

Figure 5.

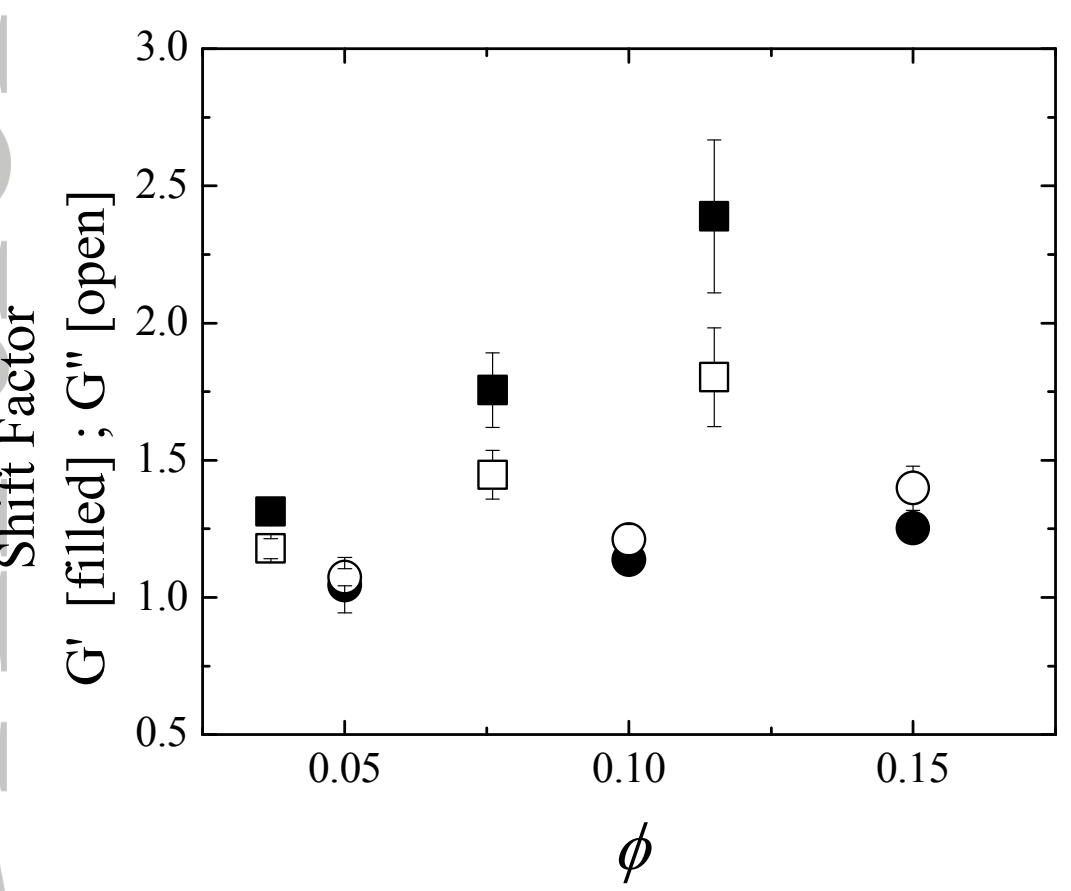

symbols) moduli onto the underlying colloidal dispersion medium for suspensions of cubic particles (squares) and non-colloidal spherical particles ${ }^{10}$ (circles).

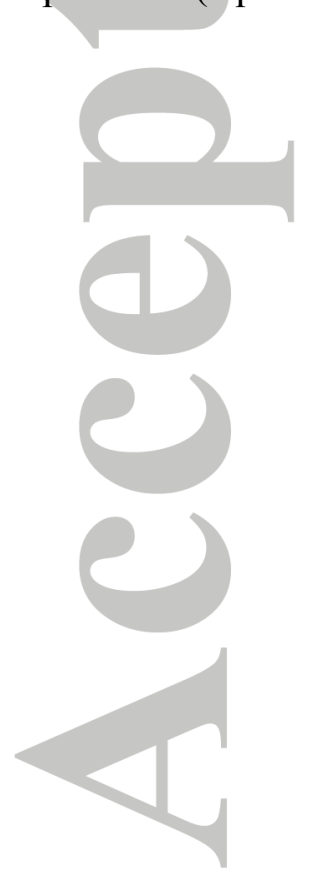



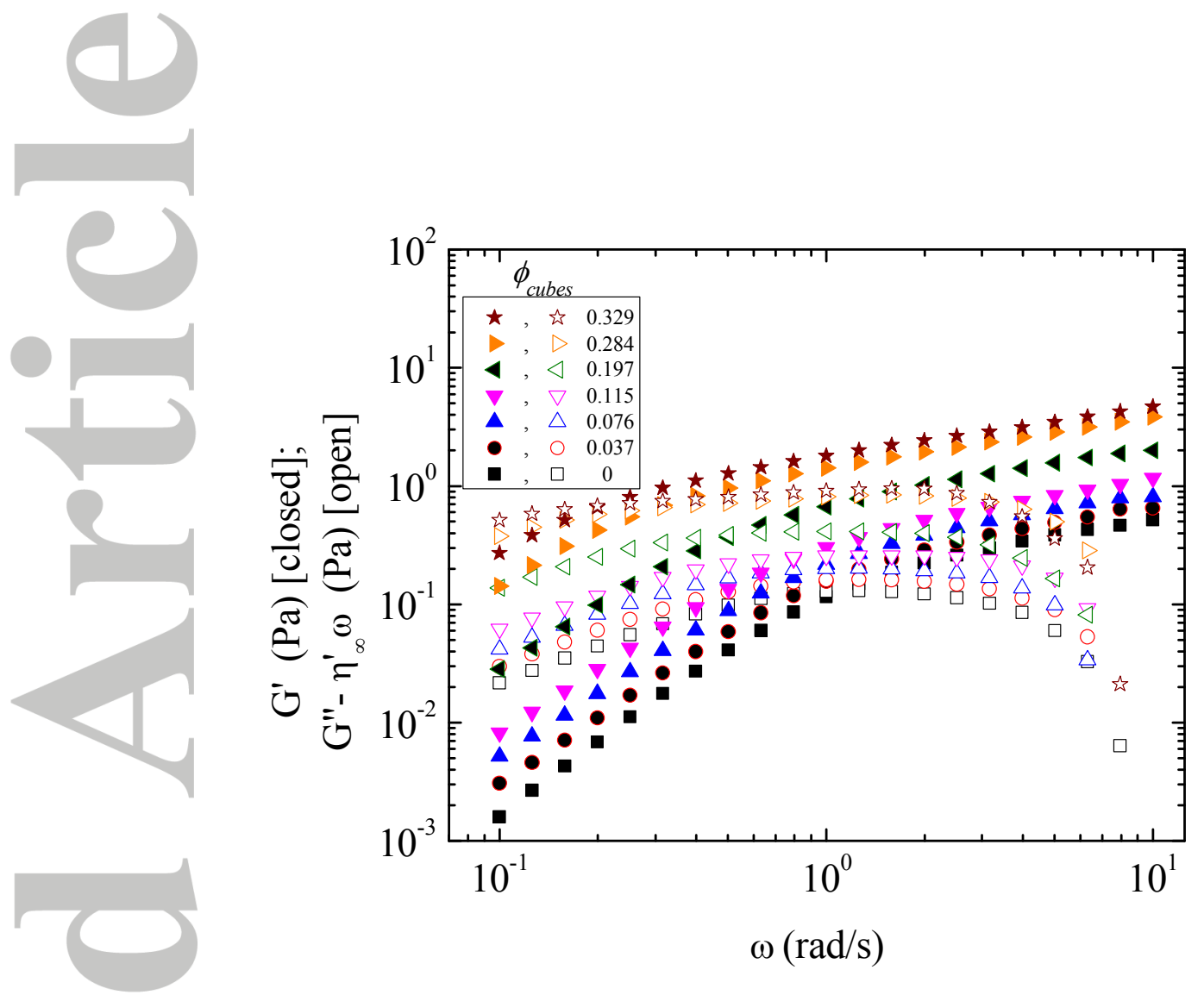

Figure 6. Storage (filled symbols) and loss moduli with the hydrodynamic component, $\eta{ }_{\infty} \omega$, subtracted off of the latter (open symbols).

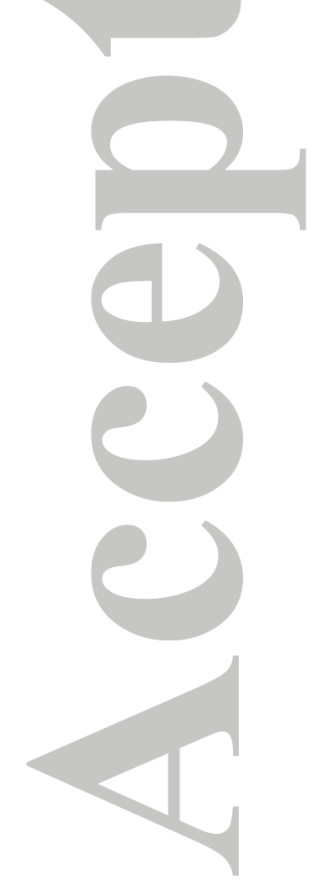




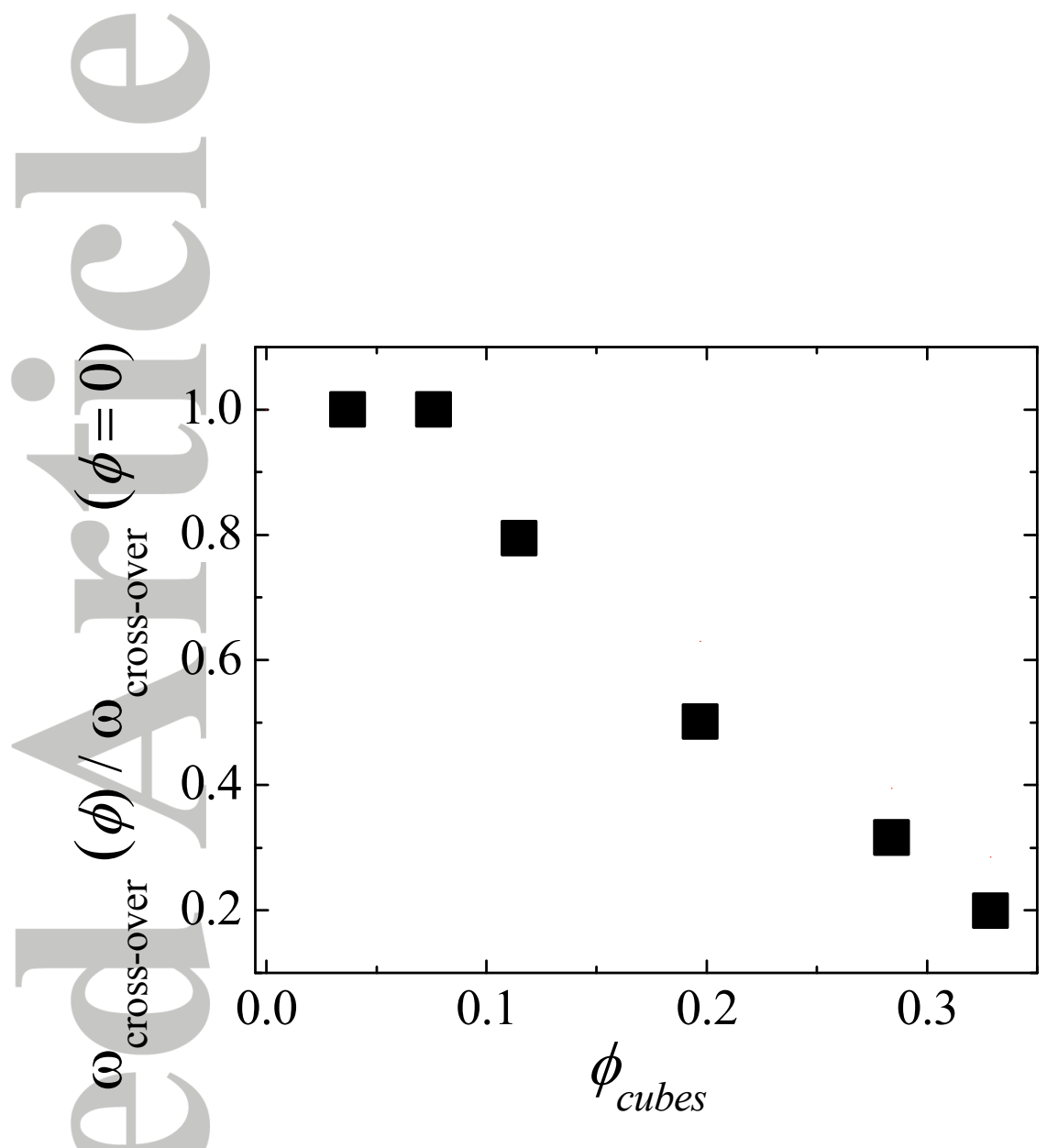

Figure 7. Suspension cross-over frequency relative to that of the underlying colloidal dispersion suspending medium as a function of the concentration of cubic particles.

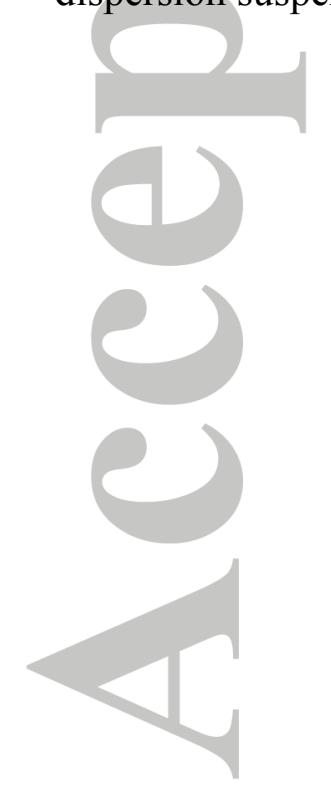



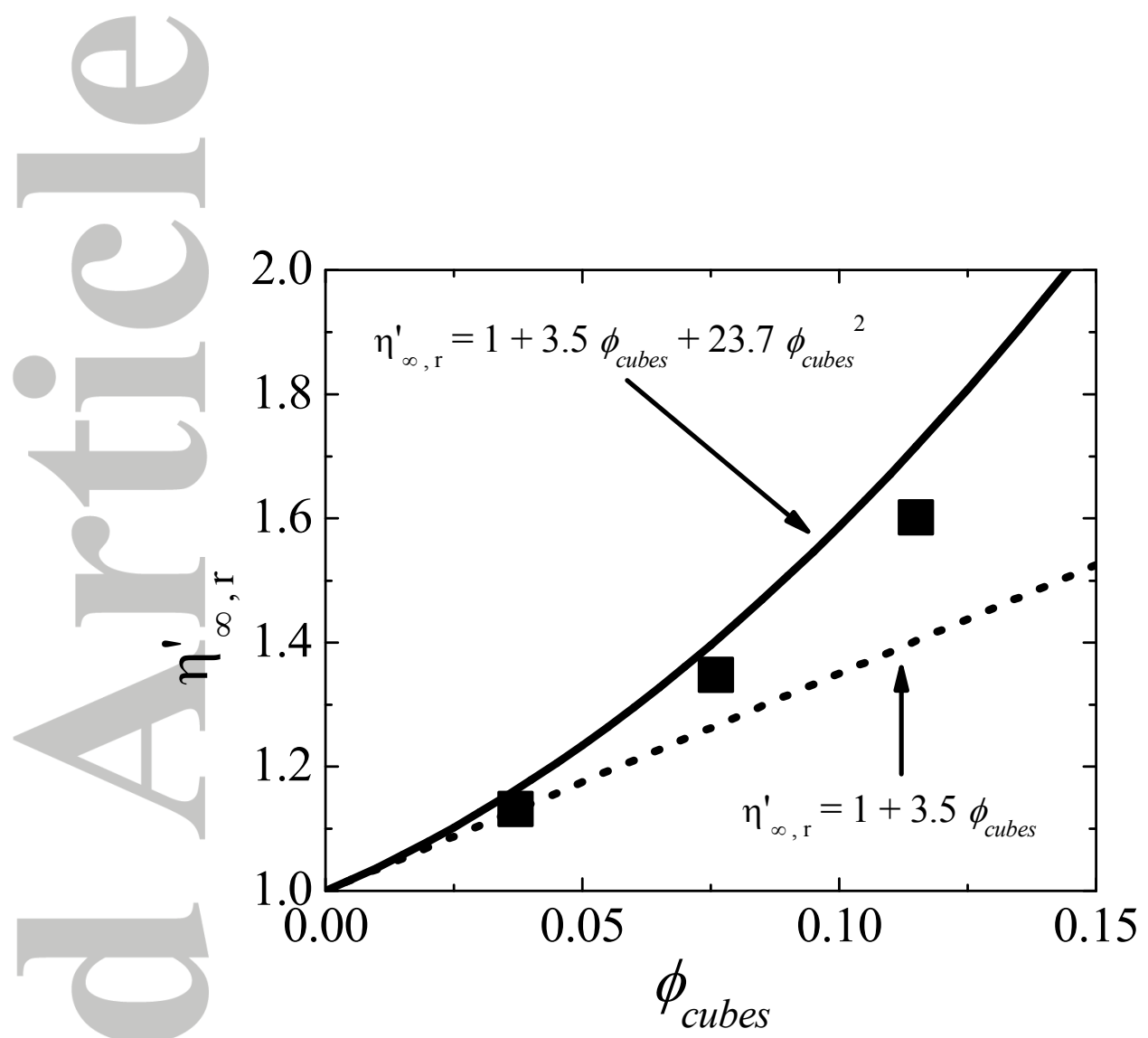

Figure 8. Relative high frequency viscosity of mixed suspensions as a function of the cubic particle volume fraction. The dashed line is the dilute limit prediction for cubic particles suspended in a Newtonian fluid and the solid line is the fit to the experimental data in the semidilute regime from Cwalina et $a l .^{22}$ Error bars are smaller than data points. 

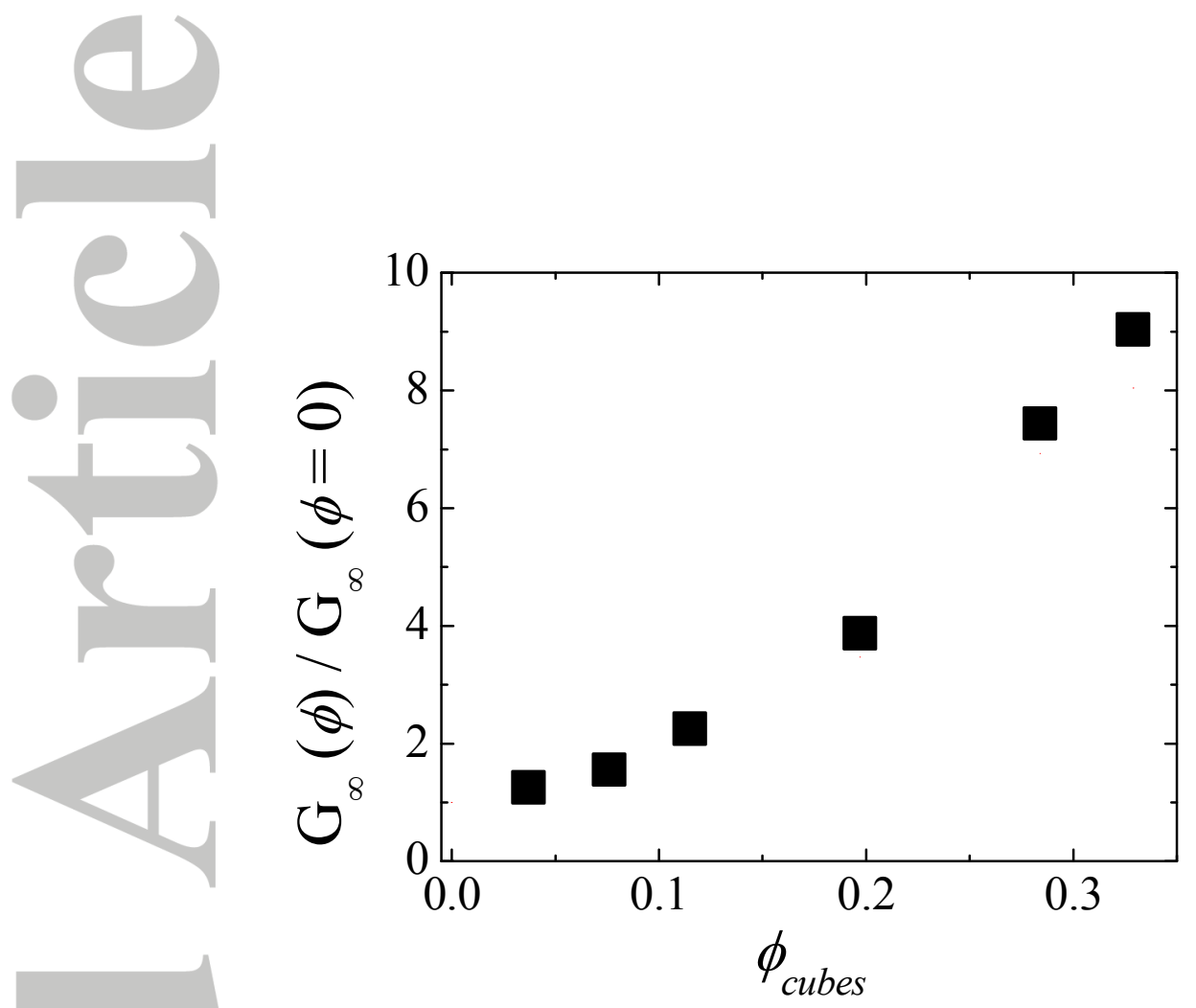

Figure 9. Plateau storage modulus relative to that of the underlying colloidal dispersion medium as a function of the concentration of cubic particles.

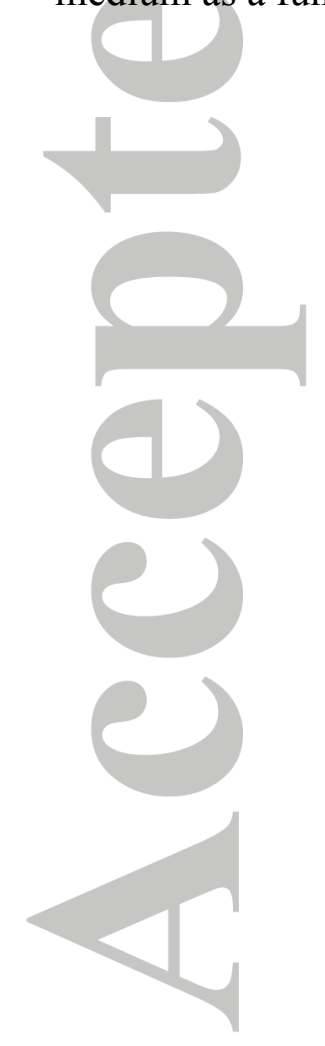



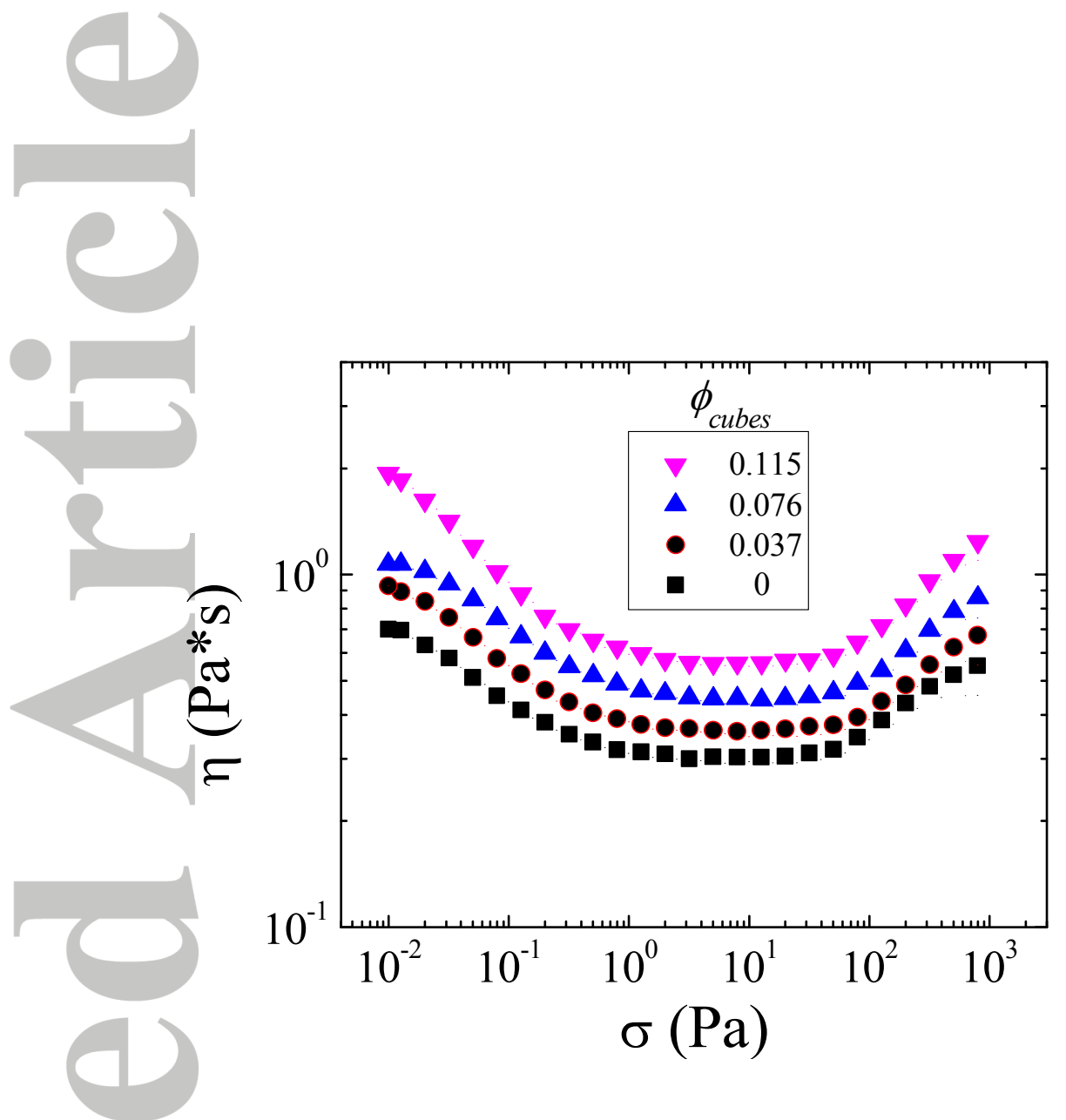

Figure 10. Steady shear viscosity of mixed suspensions of cubic particles with varying volume fraction in a concentrated colloidal dispersion as a function of the shear stress.

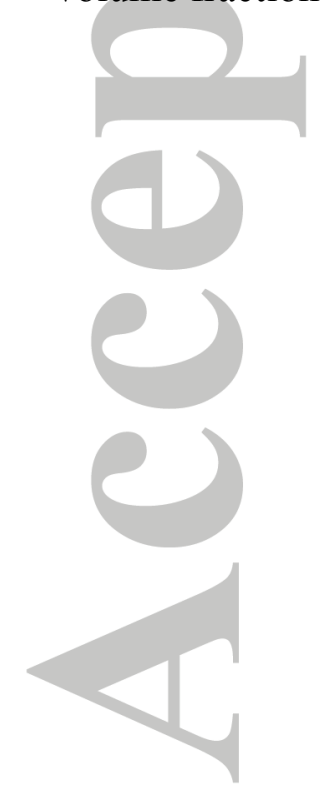




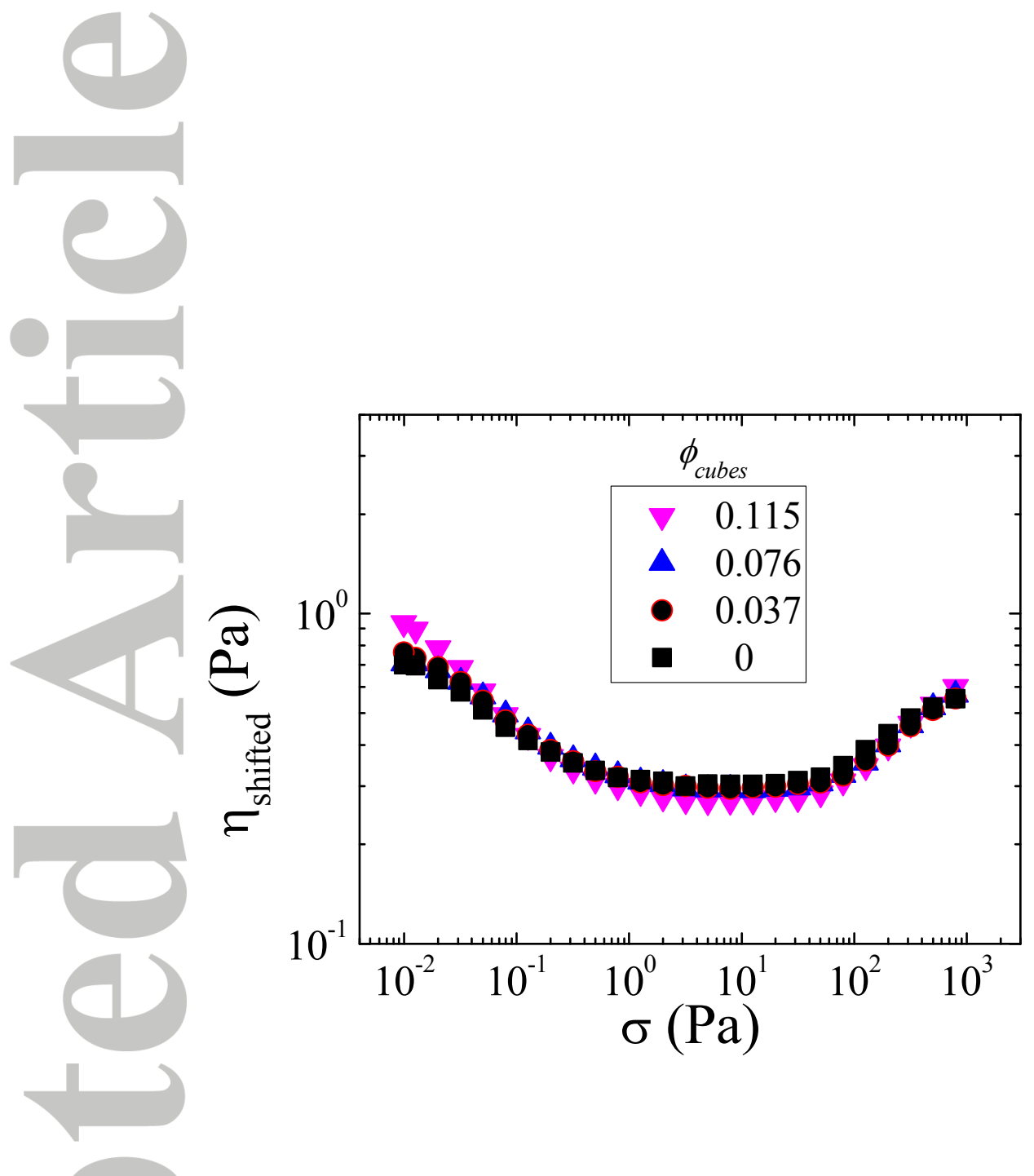

Figure 11. Suspension viscosity curves from Fig. 10 shifted onto that of the underlying colloidal dispersion medium.

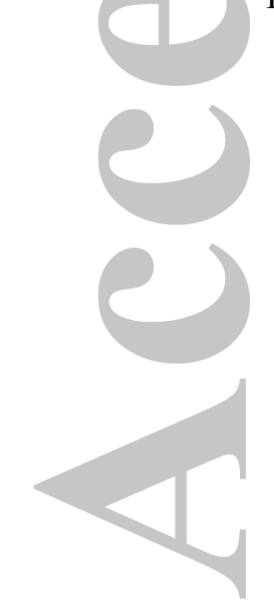

AIChE Journal

This article is protected by copvright. All rights reserved. 


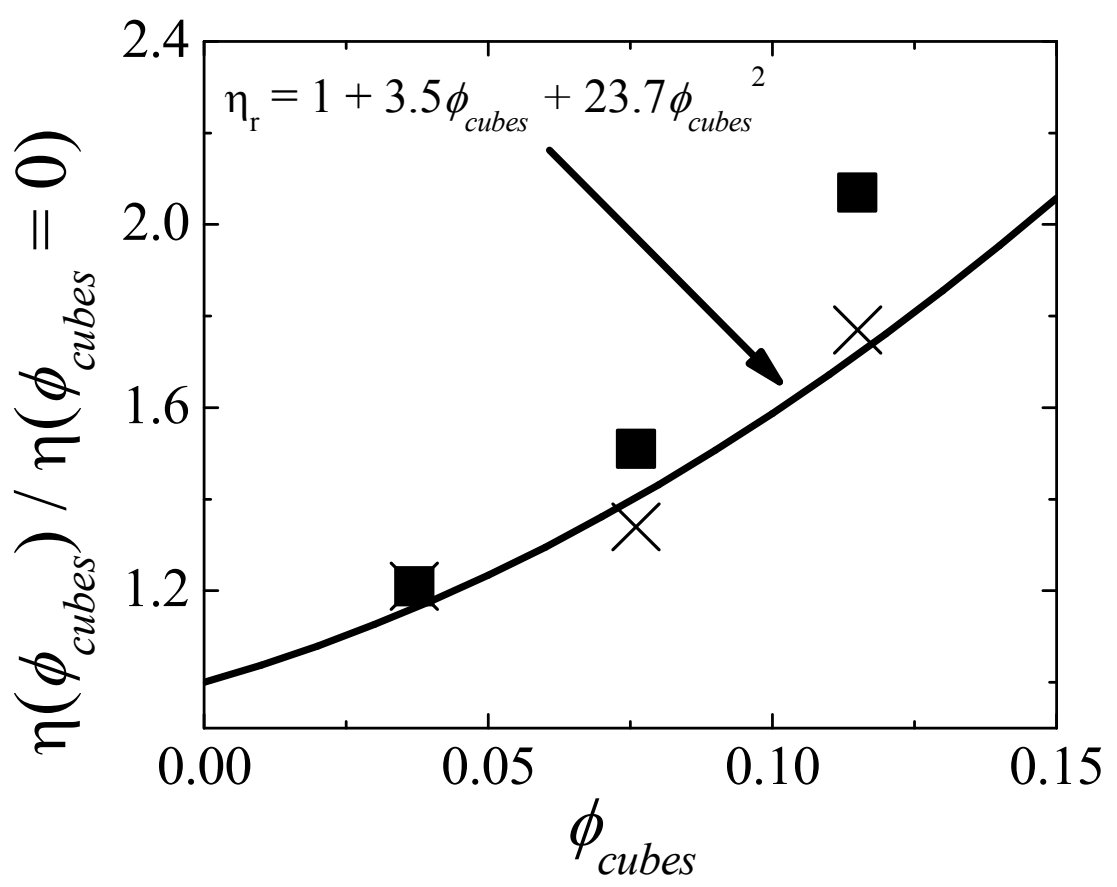

Figure 12. Measured relative steady shear viscosity of cubic particles suspended in a concentrated colloidal dispersion medium as a function of the cubic particle volume fraction.

The solid line is the concentration dependence of the suspension relative viscosity from Cwalina et $a .^{22}$ for the cubic particles in a Newtonian suspending fluid. The X's indicate the suspensions' relative viscosities where confinement effects are taken into account in determining the viscosity of the suspending colloidal dispersion. The magnitude of the viscosity increase of the colloidal dispersion is estimated from the calculations of Swan and Brady ${ }^{36}$. 

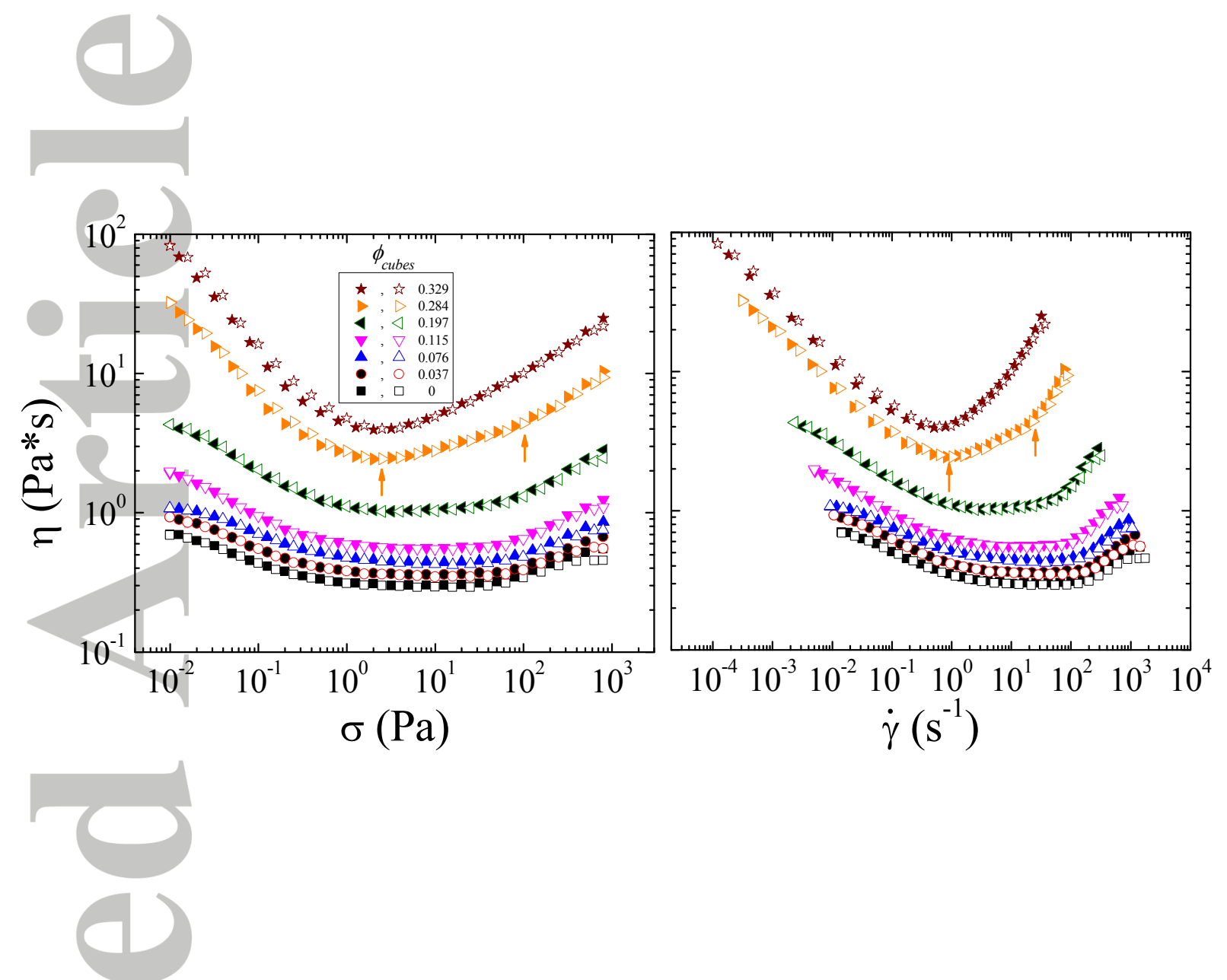

Figure 13. Steady shear viscosity of cubic particles suspended in a concentrated colloidal dispersion medium over a broad range of volume fractions plotted as a function of the shear stress (left) and shear rate (right). Open and closed symbols mark steady shear sweeps performed in both ascending and descending directions. The set of arrows in each plot is meant to guide the eye to the same two data points for the $\phi=0.284$ suspension where two distinct shear thickening transitions are evident. 


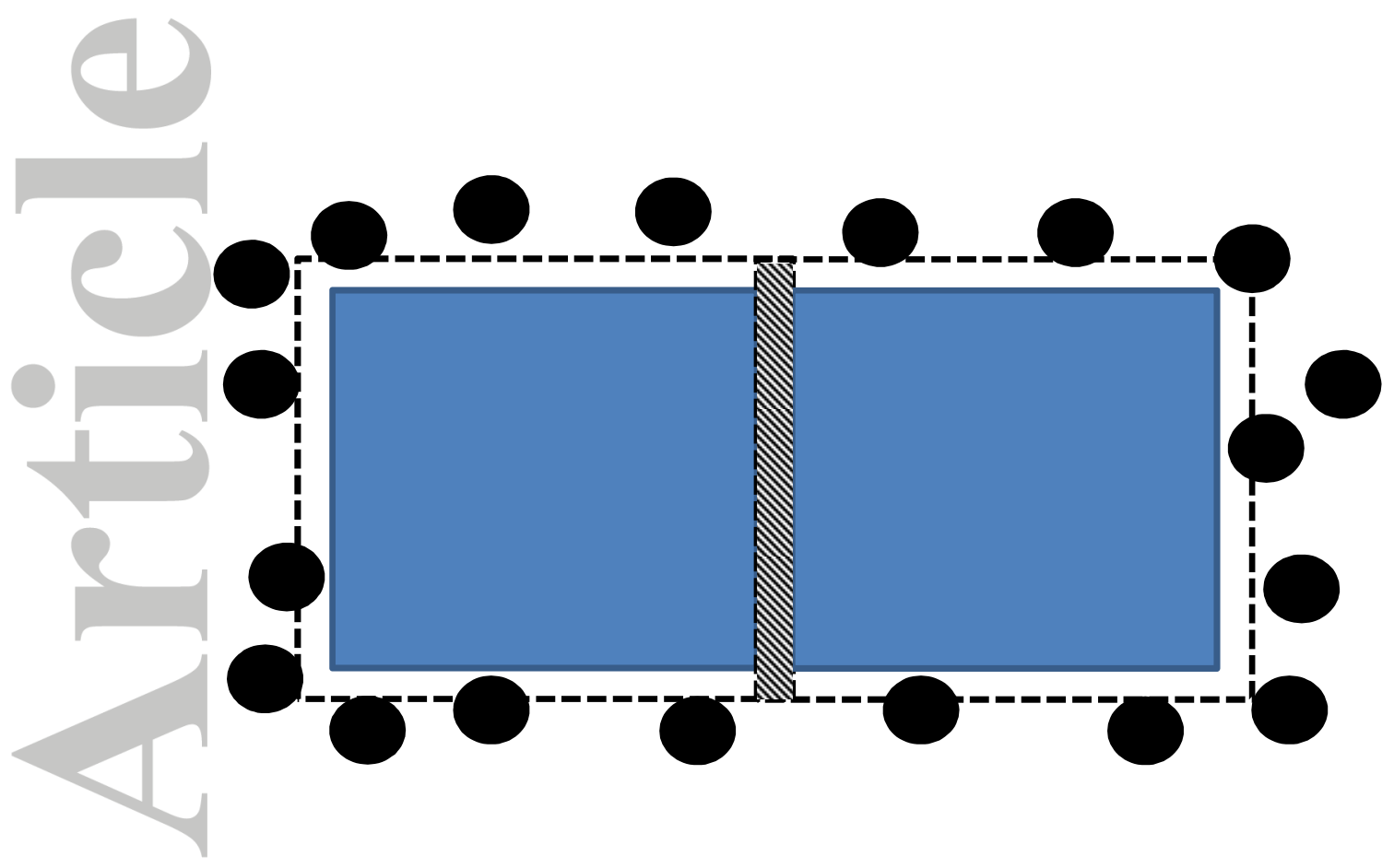

Figure 14. A to-scale illustration of $3 \mu \mathrm{m}$ edge length cubic particles and $520 \mathrm{~nm}$ colloidal spheres. The dashed line marks the boundary of the excluded region for colloidal particles based on their center location. The shaded region in between the cubes is the overlap of the regions of excluded volume used to estimate possible depletion attractions.

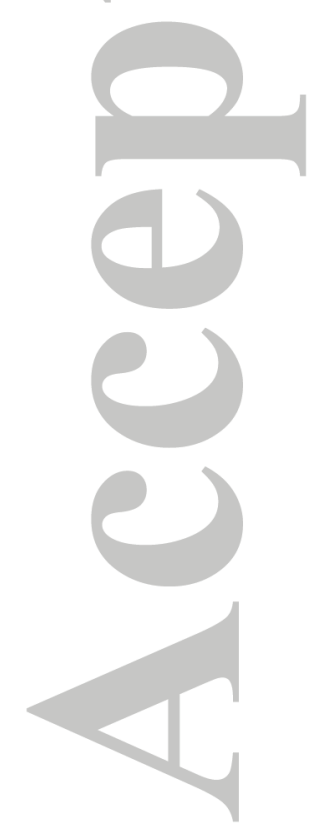




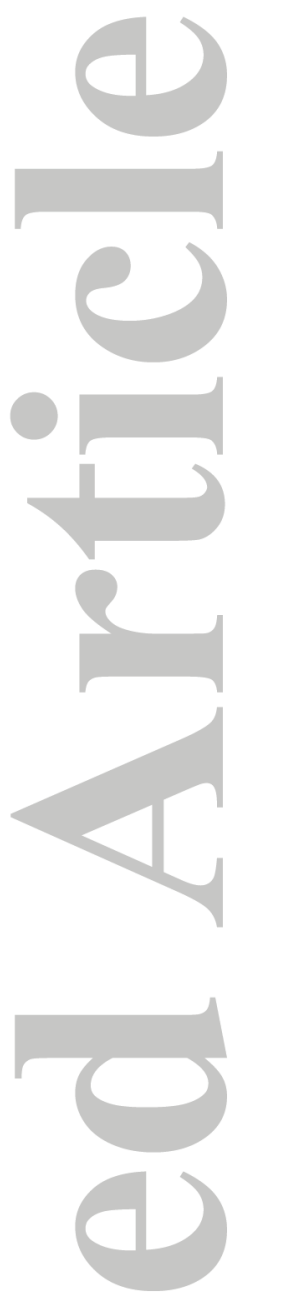

Figure 15.

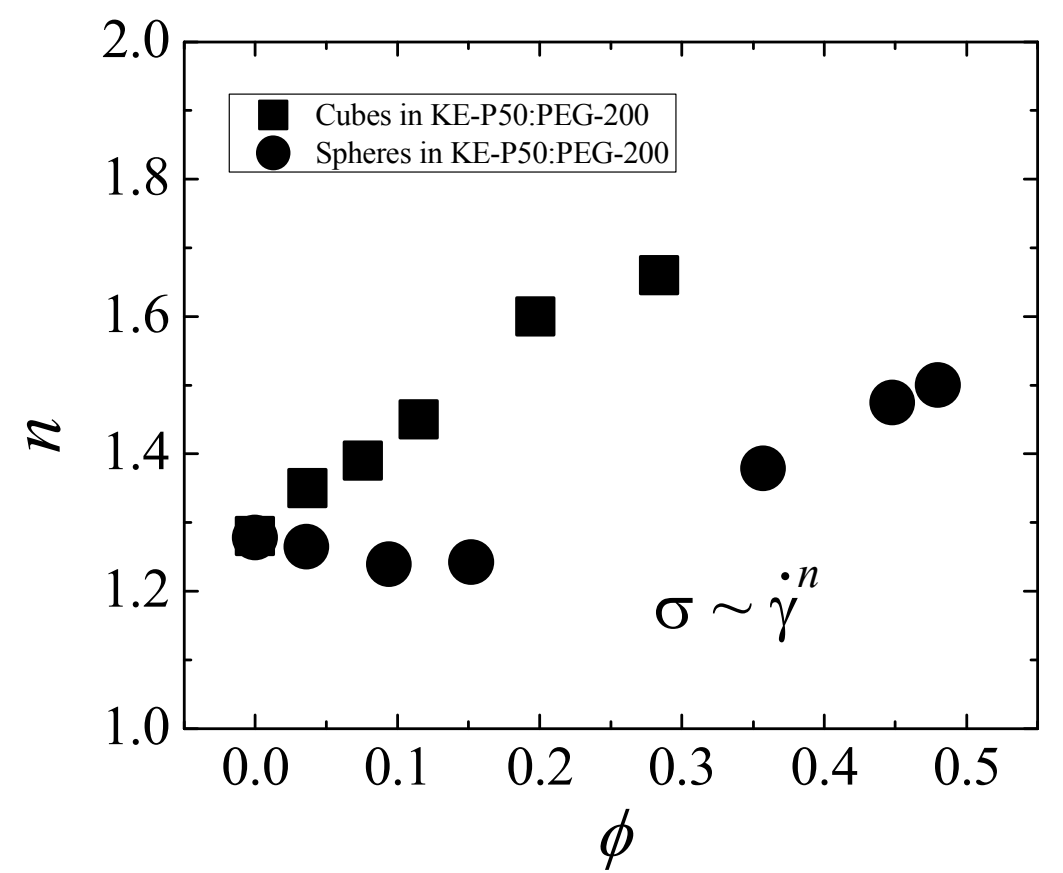

Shear thickening power law exponent, $n$, as a function of the particle volume fraction for the cubic particles suspended in a concentrated colloidal dispersion medium (squares) and for the suspensions of Cwalina and Wagner ${ }^{10}$ consisting of spherical non-colloidal particles suspended in the same concentrated colloidal dispersion medium (circles). 


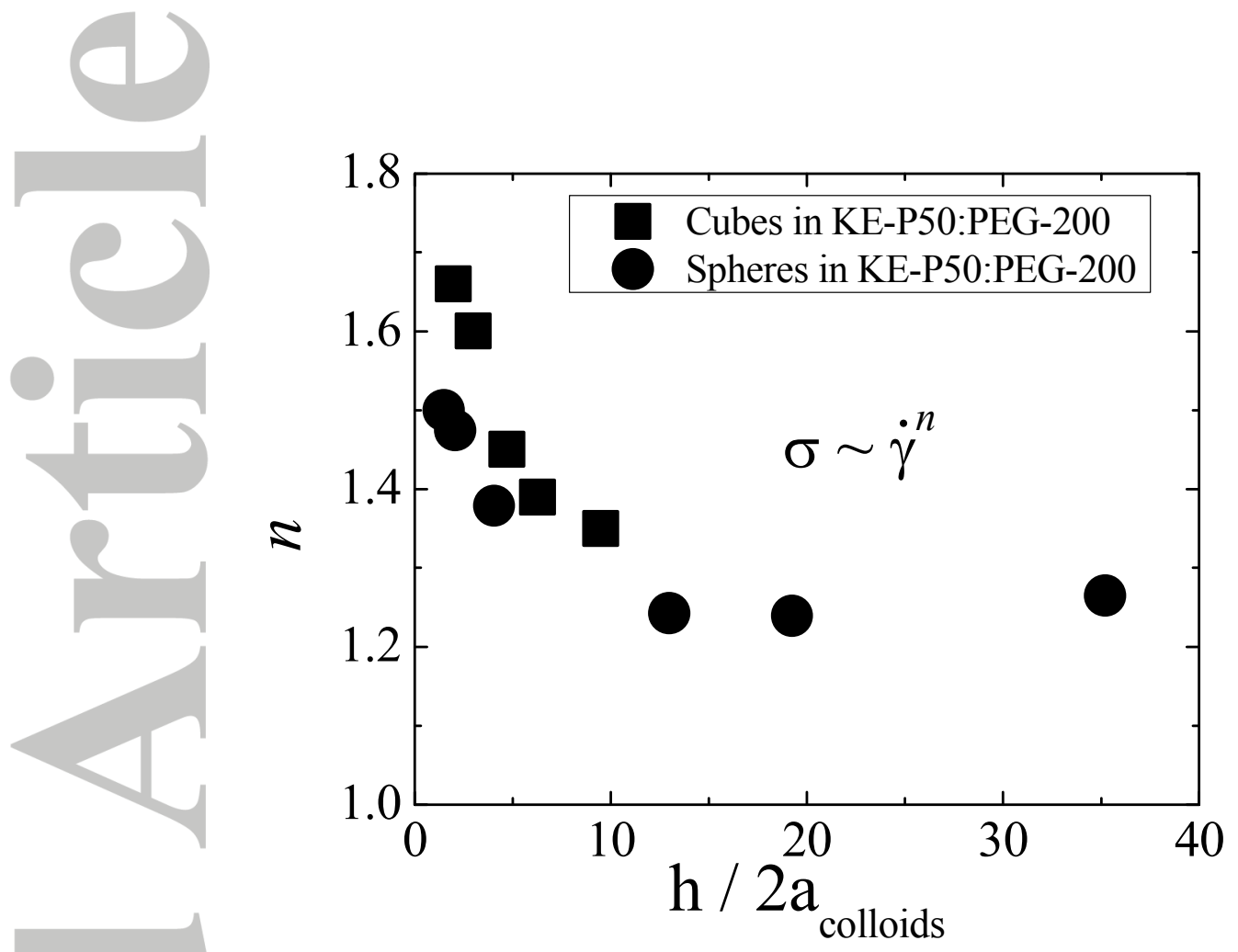

Figure 16. Shear thickening power law exponent, $n$, as a function of the number of colloidal particles spanning the characteristic gap between particle surfaces for cubic particles suspended in a concentrated colloidal dispersion medium (squares) and for the suspensions of Cwalina and Wagner ${ }^{10}$ consisting of spherical colloidal particles suspended in the same concentrated colloidal dispersion medium (circles). 


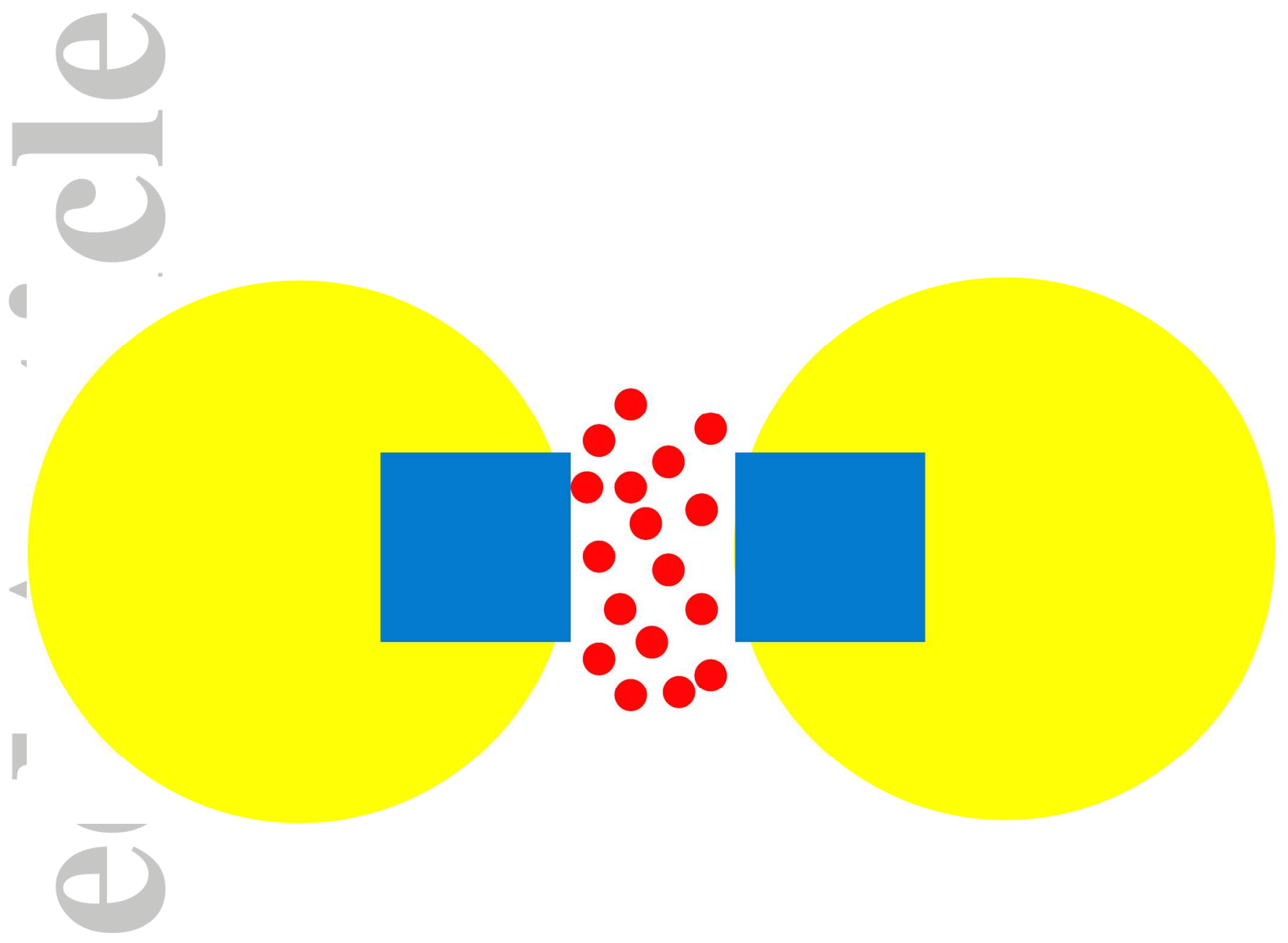

Figure 17. A to-scale representation of $520 \mathrm{~nm}$ colloidal spheres (red), $3 \mu \mathrm{m}$ edge length cubes (blue), and $10 \mu \mathrm{m}$ diameter spheres (yellow).

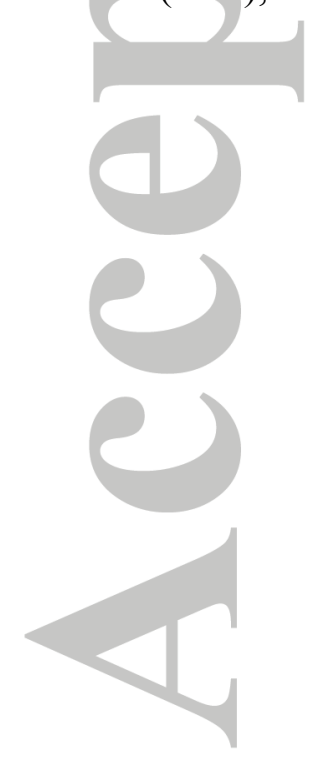



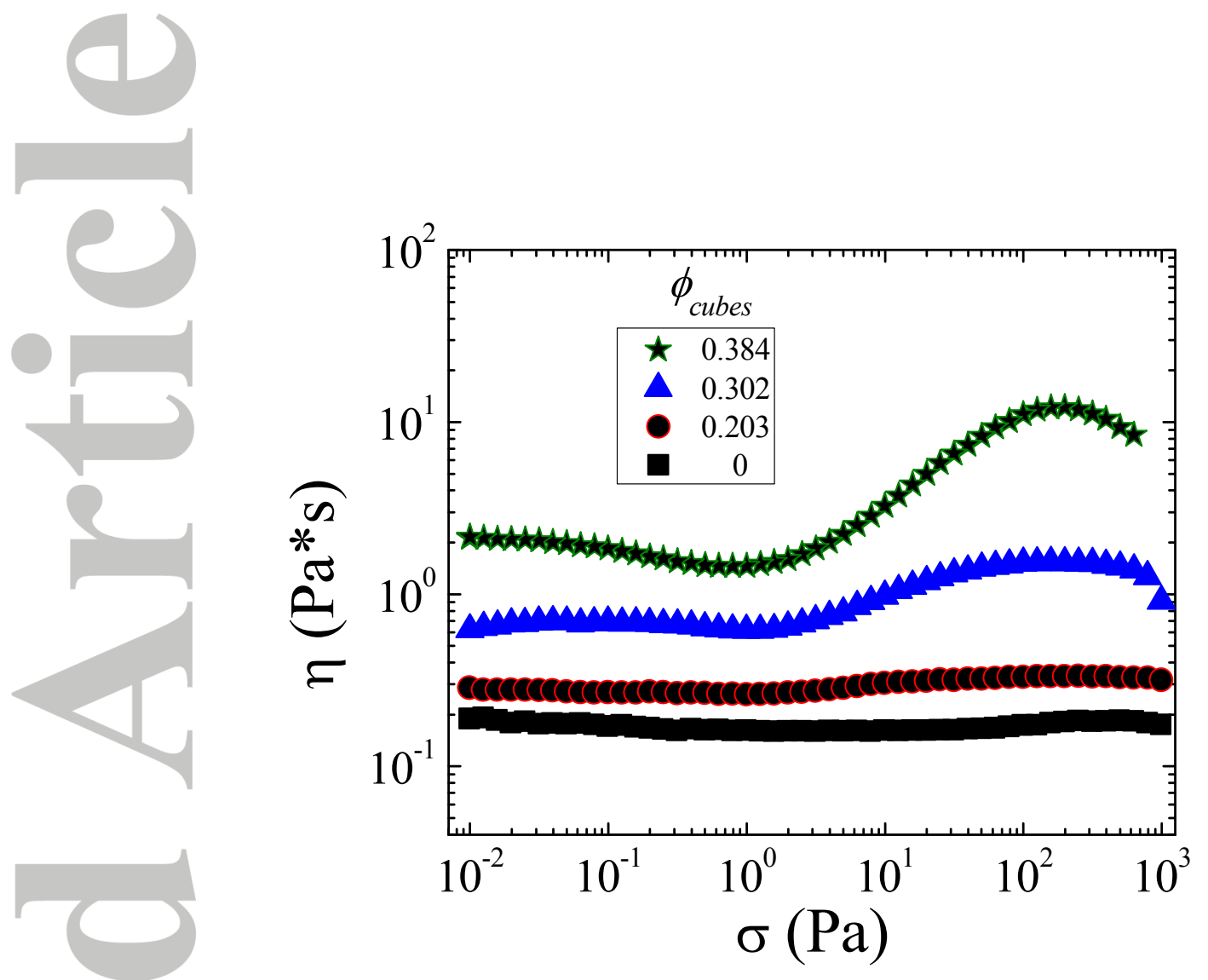

Figure 18. Steady shear rheology as a function of the applied shear stress for cubic particles suspended in a colloidal dispersion medium with a colloidal particle volume fraction of $\phi=0.20$. 


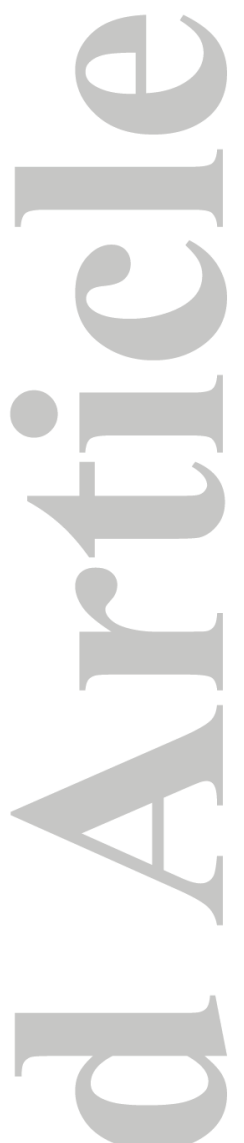

Figure 19.

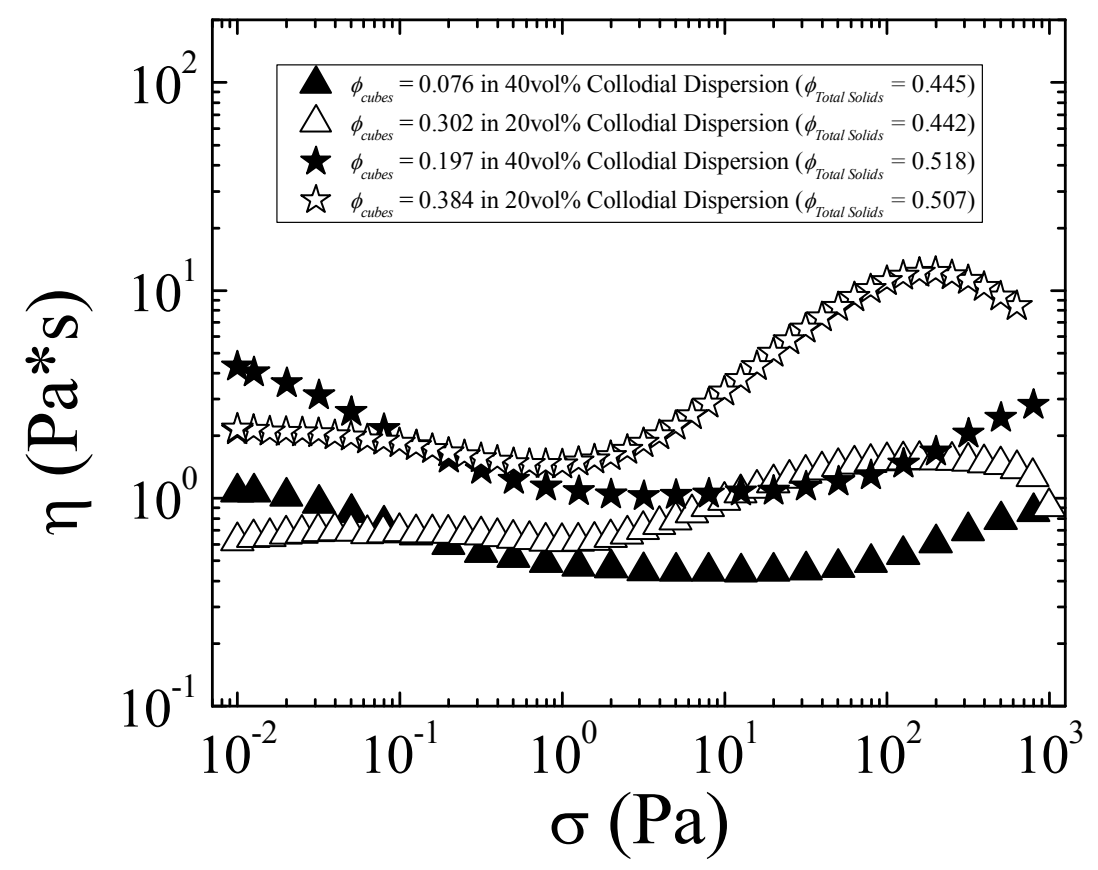

spherical colloids at nearly identical total solids loadings.

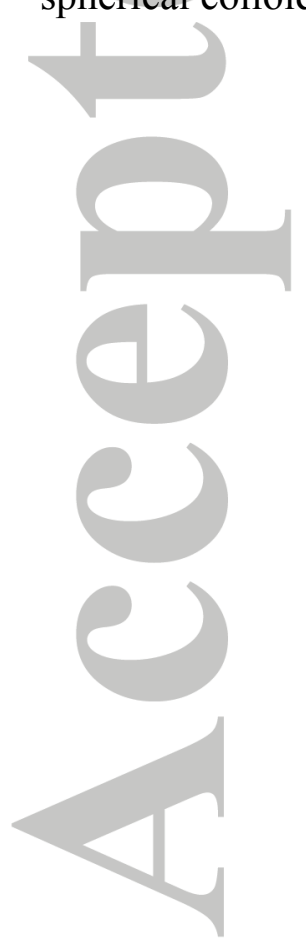

Comparison of the steady shear viscosity for suspensions of cubic particles and 


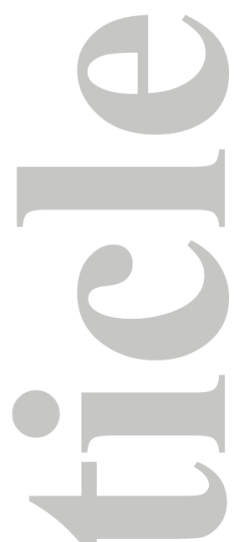

Table 1. Compositions of suspensions in this study with a colloidal dispersion suspending medium at a colloidal particle volume fraction of $\phi=0.40$.

\begin{tabular}{|c|c|c|c|c|c|}
\hline Suspension & WT\% Cubes & $\boldsymbol{\rho}_{\text {suspension }}\left(\mathbf{g} / \mathbf{c m}^{\mathbf{3}}\right)$ & $\boldsymbol{\phi}_{\text {cubes }}$ & $\boldsymbol{\phi}_{\text {Total Solids }}$ \\
\hline 1 & 5.4 & 1.49 & 0.037 & 0.422 \\
\hline 2 & 10.8 & 1.51 & 0.076 & 0.445 \\
\hline 3 & 16.1 & 1.54 & 0.115 & 0.469 \\
\hline 4 & 26.6 & 1.60 & 0.197 & 0.518 \\
\hline 5 & 37.0 & & & 0.284 & 0.570 \\
\hline & & & & & \\
\hline
\end{tabular}

AIChE Journal

This article is protected by copvright. All rights reserved. 
Table 2. Compositions of suspensions in this study with a colloidal dispersion suspending medium at a colloidal particle volume fraction of $\phi=0.20$.

\begin{tabular}{|c|c|c|c|c|}
\hline Suspension & WT\% Cubes & $\boldsymbol{\rho}_{\text {suspension }}\left(\mathbf{g} / \mathbf{c m}^{\mathbf{3}}\right)$ & $\boldsymbol{\phi}_{\text {cubes }}$ & $\boldsymbol{\phi}_{\text {Total Solids }}$ \\
\hline 7 & 30.0 & 1.47 & 0.203 & 0.362 \\
\hline 8 & 42.0 & 1.55 & 0.302 & 0.442 \\
\hline 9 & 51.1 & 1.62 & 0.384 & 0.507 \\
\hline
\end{tabular}

AIChE Journal

This article is protected by copvright. All rights reserved. 